\title{
CoNLL 2018 Shared Task: Multilingual Parsing from Raw Text to Universal Dependencies
}

\author{
Daniel Zeman ${ }^{1}$, Jan Hajič ${ }^{1}$, Martin Popel ${ }^{1}$, Martin Potthast ${ }^{2}$, \\ Milan Straka ${ }^{1}$, Filip Ginter ${ }^{3}$, Joakim Nivre ${ }^{4}$, and Slav Petrov ${ }^{5}$ \\ ${ }^{1}$ Charles University, Faculty of Mathematics and Physics \\ ${ }^{2}$ Universität Leipzig, ${ }^{3}$ University of Turku \\ ${ }^{4}$ Uppsala University, ${ }^{5}$ Google AI Language \\ \{zeman|hajic|popel|straka\}@ufal.mff.cuni.cz, \\ martin.potthast@uni-leipzig.de, figint@utu.fi, \\ joakim.nivredlingfil.uu.se, slavegoogle.com
}

\begin{abstract}
Every year, the Conference on Computational Natural Language Learning (CoNLL) features a shared task, in which participants train and test their learning systems on the same data sets. In 2018, one of two tasks was devoted to learning dependency parsers for a large number of languages, in a real-world setting without any gold-standard annotation on the input. All test sets followed the unified annotation scheme of Universal Dependencies (Nivre et al., 2016). This shared task constitutes a $2^{\text {nd }}$ edition-the first one took place in 2017 (Zeman et al., 2017); the main metric from 2017 was kept, allowing for easy comparison, and two new main metrics were introduced. New datasets added to the Universal Dependencies collection between mid-2017 and the spring of 2018 contributed to the increased difficulty of the task this year. In this overview paper, we define the task and the updated evaluation methodology, describe data preparation, report and analyze the main results, and provide a brief categorization of the different approaches of the participating systems.
\end{abstract}

\section{Introduction}

The 2017 CoNLL shared task on universal dependency parsing (Zeman et al., 2017) picked up the thread from the influential shared tasks in 2006 and 2007 (Buchholz and Marsi, 2006; Nivre et al., 2007) and evolved it in two ways: (1) the parsing process started from raw text rather than gold standard tokenization and part-of-speech tagging, and (2) the syntactic representations were consistent across languages thanks to the Universal Dependencies framework (Nivre et al., 2016). The 2018 CoNLL shared task on universal dependency parsing starts from the same premises but adds a focus on morphological analysis as well as data from new languages.

Like last year, participating systems minimally had to find labeled syntactic dependencies between words, i.e., a syntactic head for each word, and a label classifying the type of the dependency relation. In addition, this year's task featured new metrics that also scored a system's capacity to predict a morphological analysis of each word, including a part-of-speech tag, morphological features, and a lemma. Regardless of metric, the assumption was that the input should be raw text, with no gold-standard word or sentence segmentation, and no gold-standard morphological annotation. However, for teams who wanted to concentrate on one or more subtasks, segmentation and morphology predicted by the baseline UDPipe system (Straka et al., 2016) was made available just like last year.

There are eight new languages this year: Afrikaans, Armenian, Breton, Faroese, Naija, Old French, Serbian, and Thai; see Section 2 for more details. The two new evaluation metrics are described in Section 3. 


\section{Data}

In general, we wanted the participating systems to be able to use any data that is available free of charge for research and educational purposes (so that follow-up research is not obstructed). We deliberately did not place upper bounds on data sizes (in contrast to e.g. Nivre et al. (2007)), despite the fact that processing large amounts of data may be difficult for some teams. Our primary objective was to determine the capability of current parsers provided with large amounts of freely available data.

In practice, the task was formally closed, i.e., we listed the approved data resources so that all participants were aware of their options. However, the selection was rather broad, ranging from Wikipedia dumps over the OPUS parallel corpora (Tiedemann, 2012) to morphological transducers. Some of the resources were proposed by the participating teams.

We provided dependency-annotated training and test data, and also large quantities of crawled raw texts. Other language resources are available from third-party servers and we only referred to the respective download sites.

\subsection{Training Data: UD 2.2}

Training and development data came from the Universal Dependencies (UD) 2.2 collection (Nivre et al., 2018). This year, the official UD release immediately followed the test phase of the shared task. The training and development data were available to the participating teams as a prerelease; these treebanks were then released exactly in the state in which they appeared in the task. ${ }^{1}$ The participants were instructed to only use the UD data from the package released for the shared task. In theory, they could locate the (yet unreleased) test data in the development repositories on GitHub, but they were trusted that they would not attempt to do so.

82 UD treebanks in 57 languages were included in the shared task $;^{2}$ however, nine of the smaller treebanks consisted solely of test data, with no data at all or just a few sentences available for training. 16 languages had two or more treebanks

${ }^{1}$ UD 2.2 also contains other treebanks that were not included in the task for various reasons, and that may have been further developed even during the duration of the task.

${ }^{2}$ Compare with the 81 treebanks and 49 languages in the 2017 task. from different sources, often also from different domains. ${ }^{3}$ See Table 1 for an overview.

61 treebanks contain designated development data. Participants were asked not to use it for training proper but only for evaluation, development, tuning hyperparameters, doing error analysis etc. Seven treebanks have reasonablysized training data but no development data; only two of them, Irish and North Sámi, are the sole treebanks of their respective languages. For those treebanks cross-validation had to be used during development, but the entire dataset could be used for training once hyperparameters were determined. Five treebanks consist of extra test sets: they have no training or development data of their own, but large training data exist in other treebanks of the same languages (Czech-PUD, English-PUD, FinnishPUD, Japanese-Modern and Swedish-PUD, respectively). The remaining nine treebanks are low-resource languages. Their "training data" was either a tiny sample of a few dozen sentences (Armenian, Buryat, Kazakh, Kurmanji, Upper Sorbian), or there was no training data at all (Breton, Faroese, Naija, Thai). Unlike in the 2017 task, these languages were not "surprise languages", that is, the participants knew well in advance what languages to expect. The last two languages are particularly difficult: Naija is a pidgin spoken in Nigeria; while it can be expected to bear some similarity to English, its spelling is significantly different from standard English, and no resources were available to learn it. Even harder was Thai with a writing system that does not separate words by spaces; the Facebook word vectors were probably the only resource among the approved additional data where participants could learn something about words in Thai (Rosa and Mareček, 2018; Smith et al., 2018). It was also possible to exploit the fact that there is a 1-1 sentence mapping between the Thai test set and the other four PUD test sets. ${ }^{4}$

Participants received the training and development data with gold-standard tokenization, sentence segmentation, POS tags and dependency re-

\footnotetext{
${ }^{3}$ We distinguish treebanks of the same language by their short names or acronyms. Hence, the two treebanks of Ancient Greek are identified as Perseus and PROIEL, the three treebanks of Latin are ITTB, Perseus and PROIEL, etc.

${ }^{4}$ While the test datasets were not available to the teams when they developed their systems, the documentation of the treebanks was supplied together with the training data, hence the teams could learn that the PUD treebanks were parallel.
} 


\begin{tabular}{|c|c|c|c|}
\hline Language & Tbk Code & 2017 & TrWrds \\
\hline Afrikaans & af_afribooms & NA & $34 \mathrm{~K}$ \\
\hline Ancient Greek & grc_perseus & grc & $160 \mathrm{~K}$ \\
\hline Ancient Greek & grc_proiel & grc_proiel & $187 \mathrm{~K}$ \\
\hline Arabic & ar_padt & ar & $224 \mathrm{~K}$ \\
\hline Armenian & hy_armtdp & NA & $1 \mathrm{~K}$ \\
\hline Basque & eu_bdt & eu & $73 \mathrm{~K}$ \\
\hline Breton & br_keb & NA & $0 \mathrm{~K}$ \\
\hline Bulgarian & bg_btb & bg & $124 \mathrm{~K}$ \\
\hline Buryat & bxr_bdt & bxr & $0 \mathrm{~K}$ \\
\hline Catalan & ca_ancora & $\mathrm{ca}$ & $418 \mathrm{~K}$ \\
\hline Chinese & zh_gsd & $\mathrm{zh}$ & $97 \mathrm{~K}$ \\
\hline Croatian & hr_set & hr & $154 \mathrm{~K}$ \\
\hline Czech & cs_cac & cs_cac & $473 \mathrm{~K}$ \\
\hline Czech & cs_fictree & NA & $134 \mathrm{~K}$ \\
\hline Czech & cs_pdt & cs & $1,173 \mathrm{~K}$ \\
\hline Czech & cs_pud & cs_pud & $0 \mathrm{~K}$ \\
\hline Danish & da_ddt & da & $80 \mathrm{~K}$ \\
\hline Dutch & nl_alpino & $\mathrm{nl}$ & $186 \mathrm{~K}$ \\
\hline Dutch & nl_lassysmall & nl_lassysmall & $75 \mathrm{~K}$ \\
\hline English & en_ewt & en & $205 \mathrm{~K}$ \\
\hline English & en_gum & NA & $54 \mathrm{~K}$ \\
\hline English & en_lines & en_lines & $50 \mathrm{~K}$ \\
\hline English & en_pud & en_pud & $0 \mathrm{~K}$ \\
\hline Estonian & et_edt & et & $288 \mathrm{~K}$ \\
\hline Faroese & fo_oft & NA & $0 \mathrm{~K}$ \\
\hline Finnish & fi_ftb & fi_ftb & $128 \mathrm{~K}$ \\
\hline Finnish & fi_pud & fi_pud & $0 \mathrm{~K}$ \\
\hline Finnish & fi_tdt & fi & $163 \mathrm{~K}$ \\
\hline French & fr_gsd & fr & $357 \mathrm{~K}$ \\
\hline French & fr_sequoia & fr_sequoia & $51 \mathrm{~K}$ \\
\hline French & fr_spoken & NA & $15 \mathrm{~K}$ \\
\hline Galician & gl_ctg & $\mathrm{gl}$ & $79 \mathrm{~K}$ \\
\hline Galician & gl_treegal & gl_treegal & $15 \mathrm{~K}$ \\
\hline German & de_gsd & de & $264 \mathrm{~K}$ \\
\hline Gothic & got_proiel & got & $35 \mathrm{~K}$ \\
\hline Greek & el_gdt & el & $42 \mathrm{~K}$ \\
\hline Hebrew & he_htb & he & $138 \mathrm{~K}$ \\
\hline Hindi & hi_hdtb & hi & $281 \mathrm{~K}$ \\
\hline Hungarian & hu_szeged & hu & $20 \mathrm{~K}$ \\
\hline Indonesian & id_gsd & id & $98 \mathrm{~K}$ \\
\hline Irish & ga_idt & ga & $14 \mathrm{~K}$ \\
\hline
\end{tabular}

\begin{tabular}{|c|c|c|c|}
\hline Language & Tbk Code & 2017 & TrWrds \\
\hline Italian & it_isdt & it & $276 \mathrm{~K}$ \\
\hline Italian & it_postwita & NA & $99 \mathrm{~K}$ \\
\hline Japanese & ja_gsd & ja & $162 \mathrm{~K}$ \\
\hline Japanese & ja_modern & NA & $0 \mathrm{~K}$ \\
\hline Kazakh & kk_ktb & $\mathrm{kk}$ & $1 \mathrm{~K}$ \\
\hline Korean & ko_gsd & ko & $57 \mathrm{~K}$ \\
\hline Korean & ko_kaist & NA & $296 \mathrm{~K}$ \\
\hline Kurmanji & kmr_mg & $\mathrm{kmr}$ & $0 \mathrm{~K}$ \\
\hline Latin & la_ittb & la_ittb & $270 \mathrm{~K}$ \\
\hline Latin & la_perseus & la & $18 \mathrm{~K}$ \\
\hline Latin & la_proiel & la_proiel & $172 \mathrm{~K}$ \\
\hline Latvian & lv_lvtb & lv & $81 \mathrm{~K}$ \\
\hline Naija & pcm_nsc & NA & $0 \mathrm{~K}$ \\
\hline North Sámi & sme_giella & sme & $17 \mathrm{~K}$ \\
\hline Norwegian & no_bokmaal & no_bokmaal & $244 \mathrm{~K}$ \\
\hline Norwegian & no_nynorsk & no_nynorsk & $245 \mathrm{~K}$ \\
\hline Norwegian & no_nynorsklia & NA & $4 \mathrm{~K}$ \\
\hline Old Church Slavonic & cu_proiel & $\mathrm{cu}$ & $37 \mathrm{~K}$ \\
\hline Old French & fro_srcmf & NA & $136 \mathrm{~K}$ \\
\hline Persian & fa_seraji & fa & $121 \mathrm{~K}$ \\
\hline Polish & pl_lfg & NA & $105 \mathrm{~K}$ \\
\hline Polish & pl_sz & $\mathrm{pl}$ & $63 \mathrm{~K}$ \\
\hline Portuguese & pt_bosque & $\mathrm{pt}$ & $207 \mathrm{~K}$ \\
\hline Romanian & ro_rrt & ro & $185 \mathrm{~K}$ \\
\hline Russian & ru_syntagrus & ru_syntagrus & $872 \mathrm{~K}$ \\
\hline Russian & ru_taiga & NA & $10 \mathrm{~K}$ \\
\hline Serbian & sr_set & NA & $66 \mathrm{~K}$ \\
\hline Slovak & sk_snk & sk & $81 \mathrm{~K}$ \\
\hline Slovenian & sl_ssj & sl & $113 \mathrm{~K}$ \\
\hline Slovenian & sl_sst & sl_sst & $19 \mathrm{~K}$ \\
\hline Spanish & es_ancora & es_ancora & $445 \mathrm{~K}$ \\
\hline Swedish & sv_lines & sv_lines & $48 \mathrm{~K}$ \\
\hline Swedish & sv_pud & sv_pud & $0 \mathrm{~K}$ \\
\hline Swedish & sv_talbanken & sv & $67 \mathrm{~K}$ \\
\hline Thai & th_pud & NA & $0 \mathrm{~K}$ \\
\hline Turkish & tr_imst & $\operatorname{tr}$ & $38 \mathrm{~K}$ \\
\hline Ukrainian & uk_iu & uk & $75 \mathrm{~K}$ \\
\hline Upper Sorbian & hsb_ufal & hsb & $0 \mathrm{~K}$ \\
\hline Urdu & ur_udtb & ur & $109 \mathrm{~K}$ \\
\hline Uyghur & ug_udt & ug & $19 \mathrm{~K}$ \\
\hline Vietnamese & vi_vtb & vi & $20 \mathrm{~K}$ \\
\hline
\end{tabular}

Table 1: Overview of the 82 test treebanks. TbkCode $=$ Treebank identifier, consisting of the ISO 639 language code followed by a treebank-specific code. $\mathbf{2 0 1 7}=$ Code of the corresponding treebank in the 2017 task if applicable ("NA" otherwise). TrWrds = Size of training data, rounded to the nearest thousand words.

lations; and for most languages also lemmas and morphological features.

Cross-domain and cross-language training was allowed and encouraged. Participants were free to train models on any combination of the training treebanks and apply it to any test set.

\subsection{Supporting Data}

To enable the induction of custom embeddings and the use of semi-supervised methods in general, the participants were provided with supporting resources primarily consisting of large text corpora for many languages in the task, as well as embeddings pre-trained on these corpora. In total, 5.9 M sentences and $90 \mathrm{G}$ words in 45 languages are available in CoNLL-U format (Ginter et al., 2017); the per-language sizes of the corpus are listed in Table 2.

See Zeman et al. (2017) for more details on how the raw texts and embeddings were processed. Note that the resource was originally prepared for the 2017 task and it was not extended to include the eight new languages; however, some of the new languages are covered by the word vectors provided by Facebook (Bojanowski et al., 2016) and approved for the shared task. 


\begin{tabular}{|c|c|}
\hline Language & Words \\
\hline English (en) & $9,441 \mathrm{M}$ \\
\hline German (de) & $6,003 \mathrm{M}$ \\
\hline Portuguese (pt) & $5,900 \mathrm{M}$ \\
\hline Spanish (es) & $5,721 \mathrm{M}$ \\
\hline French (fr) & $5,242 \mathrm{M}$ \\
\hline Polish (pl) & $5,208 \mathrm{M}$ \\
\hline Indonesian (id) & $5,205 \mathrm{M}$ \\
\hline Japanese (ja) & $5,179 \mathrm{M}$ \\
\hline Italian (it) & $5,136 \mathrm{M}$ \\
\hline Vietnamese (vi) & $4,066 \mathrm{M}$ \\
\hline Turkish (tr) & $3,477 \mathrm{M}$ \\
\hline Russian (ru) & $3,201 \mathrm{M}$ \\
\hline Swedish (sv) & $2,932 \mathrm{M}$ \\
\hline Dutch (nl) & $2,914 \mathrm{M}$ \\
\hline Romanian (ro) & $2,776 \mathrm{M}$ \\
\hline Czech (cs) & $2,005 \mathrm{M}$ \\
\hline Hungarian (hu) & $1,624 \mathrm{M}$ \\
\hline Danish (da) & $1,564 \mathrm{M}$ \\
\hline Chinese (zh) & $1,530 \mathrm{M}$ \\
\hline Norwegian-Bokmål (no) & $1,305 \mathrm{M}$ \\
\hline Persian (fa) & $1,120 \mathrm{M}$ \\
\hline Finnish (fi) & $1,008 \mathrm{M}$ \\
\hline Arabic (ar) & $963 \mathrm{M}$ \\
\hline Catalan (ca) & $860 \mathrm{M}$ \\
\hline Slovak (sk) & $811 \mathrm{M}$ \\
\hline Greek (el) & $731 \mathrm{M}$ \\
\hline Hebrew (he) & $615 \mathrm{M}$ \\
\hline Croatian (hr) & $583 \mathrm{M}$ \\
\hline Ukrainian (uk) & $538 \mathrm{M}$ \\
\hline Korean (ko) & $527 \mathrm{M}$ \\
\hline Slovenian (sl) & $522 \mathrm{M}$ \\
\hline Bulgarian (bg) & $370 \mathrm{M}$ \\
\hline Estonian (et) & $328 \mathrm{M}$ \\
\hline Latvian (lv) & $276 \mathrm{M}$ \\
\hline Galician $(\mathrm{gl})$ & $262 \mathrm{M}$ \\
\hline Latin (la) & $244 \mathrm{M}$ \\
\hline Basque (eu) & $155 \mathrm{M}$ \\
\hline Hindi (hi) & $91 \mathrm{M}$ \\
\hline Norwegian-Nynorsk (no) & $76 \mathrm{M}$ \\
\hline Kazakh (kk) & $54 \mathrm{M}$ \\
\hline Urdu (ur) & $46 \mathrm{M}$ \\
\hline Irish (ga) & $24 \mathrm{M}$ \\
\hline Ancient Greek (grc) & $7 \mathrm{M}$ \\
\hline Uyghur (ug) & $3 \mathrm{M}$ \\
\hline Kurdish (kmr) & $3 \mathrm{M}$ \\
\hline Upper Sorbian (hsb) & $2 \mathrm{M}$ \\
\hline Buryat (bxr) & $413 \mathrm{~K}$ \\
\hline North Sámi (sme) & $331 \mathrm{~K}$ \\
\hline Old Church Slavonic (cu) & $28 \mathrm{~K}$ \\
\hline Total & $90,669 \mathrm{M}$ \\
\hline
\end{tabular}

Table 2: Supporting data overview: Number of words ( $\mathrm{M}=$ million; $\mathrm{K}=$ thousand) for each language.

\subsection{Test Data: UD 2.2}

Each of the 82 treebanks mentioned in Section 2.1 has a test set. Test sets from two different treebanks of one language were evaluated separately as if they were different languages. Every test set contains at least 10,000 words (including punctuation marks). UD 2.2 treebanks that were smaller than 10,000 words were excluded from the shared task. There was no upper limit on the test data; the largest treebank had a test set comprising $170 \mathrm{~K}$ words. The test sets were officially released as a part of UD 2.2 immediately after the shared task. ${ }^{5}$

\section{Evaluation Metrics}

There are three main evaluation scores, dubbed LAS, MLAS and BLEX. All three metrics reflect word segmentation and relations between content words. LAS is identical to the main metric of the 2017 task, allowing for easy comparison; the other two metrics include part-of-speech tags, morphological features and lemmas. Participants who wanted to decrease task complexity could concentrate on improvements in just one metric; however, all systems were evaluated with all three metrics, and participants were strongly encouraged to output all relevant annotation, even if they just copy values predicted by the baseline model.

When parsers are applied to raw text, the metric must be adjusted to the possibility that the number of nodes in gold-standard annotation and in the system output vary. Therefore, the evaluation starts with aligning system nodes and gold nodes. A dependency relation cannot be counted as correct if one of the nodes could not be aligned to a gold node. See Section 3.4 and onward for more details on alignment.

The evaluation software is a Python script that computes the three main metrics and a number of additional statistics. It is freely available for download from the shared task website. ${ }^{6}$

\subsection{LAS: Labeled Attachment Score}

The standard evaluation metric of dependency parsing is the labeled attachment score (LAS), i.e., the percentage of nodes with correctly assigned reference to the parent node, including the label (type) of the relation. For scoring purposes, only

\footnotetext{
${ }^{5}$ http://hdl .handle. net/11234/1-2837

${ }^{6}$ http: //universaldependencies.org/ conl118/conl118_ud_eval.py
} 


\begin{tabular}{|l|l|}
\hline Content & $\begin{array}{l}\text { nsubj, obj, iobj, csubj, ccomp, xcomp, obl, vocative, expl, } \\
\text { dislocated, advcl, advmod, discourse, nmod, appos, nummod, } \\
\text { acl, amod, conj, fixed, flat, compound, list, parataxis, } \\
\text { orphan, goeswith, reparandum, root, dep }\end{array}$ \\
\hline Function & aux, cop, mark, det, clf, case, cc \\
\hline Ignored & punct \\
\hline
\end{tabular}

Table 3: Universal dependency relations considered as pertaining to content words and function words, respectively, in MLAS. Content word relations are evaluated directly; words attached via functional relations are treated as features of their parent nodes.

\begin{tabular}{|l|l|}
\hline Features & $\begin{array}{l}\text { Prontype, Numtype, Poss, Reflex, Foreign, Abbr, Gender, } \\
\text { Animacy, Number, Case, Definite, Degree, VerbForm, Mood, } \\
\text { Tense, Aspect, Voice, Evident, Polarity, Person, Polite }\end{array}$ \\
\hline
\end{tabular}

Table 4: Universal features whose values are evaluated in MLAS. Any other features are ignored.

universal dependency labels were taken into account, which means that language-specific subtypes such as expl:pv (pronoun of a pronominal verb), a subtype of the universal relation expl (expletive), were truncated to expl both in the gold standard and in the system output before comparing them.

In the end-to-end evaluation of our task, LAS is re-defined as the harmonic mean $\left(F_{1}\right)$ of precision $P$ and recall $R$, where

$$
\begin{gathered}
P=\frac{\# \text { correctRelations }}{\# \text { systemNodes }} \\
R=\frac{\# \text { correctRelations }}{\# \text { goldNodes }} \\
L A S=\frac{2 P R}{P+R}
\end{gathered}
$$

Note that attachment of all nodes including punctuation is evaluated. LAS is computed separately for each of the 82 test files and a macro-average of all these scores is used to rank the systems.

\subsection{MLAS: Morphology-Aware Labeled Attachment Score}

MLAS aims at cross-linguistic comparability of the scores. It is an extension of CLAS (Nivre and Fang, 2017), which was tested experimentally in the 2017 task. CLAS focuses on dependencies between content words and disregards attachment of function words; in MLAS, function words are not ignored, but they are treated as features of content words. In addition, part-of-speech tags and morphological features are evaluated, too.
The idea behind MLAS is that function words often correspond to morphological features in other languages. Furthermore, languages with many function words (e.g., English) have longer sentences than morphologically rich languages (e.g., Finnish), hence a single error in Finnish costs the parser significantly more than an error in English according to LAS.

The core part is identical to LAS (Section 3.1): for aligned system and gold nodes, their respective parent nodes are considered; if the system parent is not aligned with the gold parent, or if the universal relation label differs, the word is not counted as correctly attached. Unlike LAS, certain types of relations (Table 3) are not evaluated directly. Words attached via such relations (in either system or gold data) are not counted as independent words. Instead, they are treated as features of the content words they belong to. Therefore, a system-produced word counts as correct if it is aligned and attached correctly, its universal POS tag and selected morphological features (Table 4) are correct, all its function words are attached correctly, and their POS tags and features are also correct. Punctuation nodes are neither content nor function words; their attachment is ignored in MLAS.

\subsection{BLEX: Bilexical Dependency Score}

BLEX is similar to MLAS in that it focuses on relations between content words. Instead of morphological features, it incorporates lemmatization in the evaluation. It is thus closer to semantic content and evaluates two aspects of UD annota- 
tion that are important for language understanding: dependencies and lexemes. The inclusion of this metric should motivate the competing teams to model lemmas, the last important piece of annotation that is not captured by the other metrics. A system that scores high in all three metrics will thus be a general-purpose language-analysis tool that tackles segmentation, morphology and surface syntax.

Computation of BLEX is analogous to LAS and MLAS. Precision and recall of correct attachments is calculated, attachment of function words and punctuation is ignored (Table 3). An attachment is correct if the parent and child nodes are aligned to the corresponding nodes in gold standard, if the universal dependency label is correct, and if the lemma of the child node is correct.

A few UD treebanks lack lemmatization (or, as in Uyghur, have lemmas only for some words and not for others). A system may still be able to predict the lemmas if it learns them in other treebanks. Such system should not be penalized just because no gold standard is available; therefore, if the gold lemma is a single underscore character (“-”), any system-produced lemma is considered correct.

\subsection{Token Alignment}

UD defines two levels of token/word segmentation. The lower level corresponds to what is usually understood as tokenization. However, unlike some popular tokenization schemes, it does not include any normalization of the non-whitespace characters. We can safely assume that any two tokenizations of a text differ only in whitespace while the remaining characters are identical. There is thus a 1-1 mapping between gold and system nonwhitespace characters, and two tokens are aligned if all their characters match.

\subsection{Syntactic Word Alignment}

The higher segmentation level is based on the notion of syntactic word. Some languages contain multi-word tokens (MWT) that are regarded as contractions of multiple syntactic words. For example, the German token zum is a contraction of the preposition $z u$ "to" and the article dem "the".

Syntactic words constitute independent nodes in dependency trees. As shown by the example, it is not required that the MWT is a pure concatenation of the participating words; the simple token alignment thus does not work when MWTs are involved. Fortunately, the CoNLL-U file format used in UD clearly marks all MWTs so we can detect them both in system output and in gold data. Whenever one or more MWTs have overlapping spans of surface character offsets, the longest common subsequence algorithm is used to align syntactic words within these spans.

\subsection{Sentence Segmentation}

Words are aligned and dependencies are evaluated in the entire file without considering sentence segmentation. Still, the accuracy of sentence boundaries has an indirect impact on attachment scores: any missing or extra sentence boundary necessarily makes one or more dependency relations incorrect.

\subsection{Invalid Output}

If a system fails to produce one of the 82 files or if the file is not valid CoNLL-U format, the score of that file (counting towards the system's macroaverage) is zero.

Formal validity is defined more leniently than for UD-released treebanks. For example, a nonexistent dependency type does not render the whole file invalid, it only costs the system one incorrect relation. However, cycles and multi-root sentences are disallowed. A file is also invalid if there are character mismatches that could make the token-alignment algorithm fail.

\subsection{Extrinsic Parser Evaluation}

The metrics described above are all intrinsic measures: they evaluate the grammatical analysis task per se, with the hope that better scores correspond to output that is more useful for downstream NLP applications. Nevertheless, such correlations are not automatically granted. We thus seek to complement our task with an extrinsic evaluation, where the output of parsing systems is exploited by applications like biological event extraction, opinion analysis and negation scope resolution.

This optional track involves English only. It is organized in collaboration with the EPE initiative, ${ }^{7}$ for details see Fares et al. (2018).

\section{TIRA: The System Submission Platform}

Similarly to our 2017 task and to some other recent CoNLL shared tasks, we employed the cloud-

\footnotetext{
${ }^{7}$ http://epe.nlpl.eu/
} 
based evaluation platform TIRA (Potthast et al., 2014), ${ }^{8}$ which implements the evaluation as a service paradigm (Hanbury et al., 2015). Instead of processing test data on their own hardware and submitting the outputs, participants submit working software. Naturally, software submissions bring about additional overhead for both organizers and participants, whereas the goal of an evaluation platform like TIRA is to reduce this overhead to a bearable level.

\subsection{Blind Evaluation}

Traditionally, evaluations in shared tasks are halfblind (the test data are shared with participants while the ground truth is withheld). TIRA enables fully blind evaluation, where the software is locked in a datalock together with the test data, its output is recorded but all communication channels to the outside are closed or tightly moderated. The participants do not even see the input to their software. This feature of TIRA was not too important in the present task, as UD data is not secret, and the participants were simply trusted that they would not exploit any knowledge of the test data they might have access to.

However, closing down all communication channels also has its downsides, since participants cannot check their running software; before the system run completes, even the task moderator does not see whether the system is really producing output and not just sitting in an endless loop. In order to alleviate this extra burden, we made two modifications compared to the previous year: 1. Participants were explicitly advised to invoke shorter runs that process only a subset of the test files. The organizers would then stitch the partial runs into one set of results. 2. Participants were able to see their scores on the test set rounded to the nearest multiple of 5\%. This way they could spot anomalies possibly caused by illselected models. The exact scores remained hidden because we did not want the participants to fine-tune their systems against the test data.

\subsection{Replicability}

It is desirable that published experiments can be re-run yielding the same results, and that the algorithms can be tested on alternative test data in the future. Ensuring both requires that a to-beevaluated software is preserved in working con-

\footnotetext{
${ }^{8}$ http://www.tira.io/
}

dition for as long as possible. TIRA supplies participants with a virtual machine, offering a range of commonly used operating systems. Once deployed and tested, the virtual machines are archived to preserve the software within.

In addition, some participants agreed to share their code so that we decided to collect the respective projects in an open source repository hosted on GitHub. ${ }^{9}$

\section{Baseline System}

We prepared a set of baseline models using UDPipe 1.2 (Straka and Straková, 2017).

The baseline models were released together with the UD 2.2 training data. For each of the 73 treebanks with non-empty training data we trained one UDPipe model, utilizing training data for training and development data for hyperparameter tuning. If a treebank had no development data, we cut $10 \%$ of the training sentences and considered it as development data for the purpose of tuning hyperparameters of the baseline model (employing only the remainder of the original training data for the actual training in that case).

In addition to the treebank-specific models, we also trained a "mixed model" on samples from all treebanks. Specifically, we utilized the first 200 training sentences of each treebank (or less in case of small treebanks) as training data, and at most 20 sentences from each treebank's development set as development data.

The baseline models, together with all information needed to replicate them (hyperparameters, the modified train-dev split where applicable, and pre-computed word embeddings for the parser) are available from http://hdl.handle. net/11234/ 1-2859.

Additionally, the released archive also contains the training and development data with predicted morphology. Morphology in development data was predicted using the baseline models, morphology in training data via "jack-knifing" (split the training set into 10 parts, train a model on 9 parts, use it to predict morphology in the tenth part, repeat for all 10 target parts). The same hyperparameters were used as those used to train the baseline model on the entire training set.

The UDPipe baseline models are able to reconstruct nearly all annotation from CoNLL-U files - they can generate segmentation, tokenization,

\footnotetext{
${ }^{9}$ https://github.com/CoNLL-UD-2018
} 


\begin{tabular}{|l|l|}
\hline $\begin{array}{c}\text { Treebank without } \\
\text { training data }\end{array}$ & \multicolumn{1}{|c|}{$\begin{array}{c}\text { Substitution } \\
\text { model }\end{array}$} \\
\hline Breton KEB & mixed model \\
\hline Czech PUD & Czech PDT \\
\hline English PUD & English EWT \\
\hline Faroese OFT & mixed model \\
\hline Finnish PUD & Finnish TDT \\
\hline Japanese Modern & Japanese GSD \\
\hline Naija NSC & mixed model \\
\hline Swedish PUD & Swedish Talbanken \\
\hline Thai PUD & mixed model \\
\hline
\end{tabular}

Table 5: Substitution models of the baseline systems for treebanks without training data.

multi-word token splitting, morphological annotation (lemmas, UPOS, XPOS and FEATS) and dependency trees. Participants were free to use any part of the model in their systems - for all test sets, we provided UDPipe processed variants in addition to raw text inputs.

Baseline UDPipe Shared Task System The shared task baseline system employs the UDPipe 1.2 baseline models. For the nine treebanks without their own training data, a substitution model according to Table 5 was used.

\section{Results}

\subsection{Official Parsing Results}

Table 6 gives the main ranking of participating systems by the LAS $F_{1}$ score macro-averaged over all 82 test files. The table also shows the performance of the baseline UDPipe system; 17 of the 25 systems managed to outperform it. The baseline is comparatively weaker than in the 2017 task (only 12 out of 32 systems beat the baseline there). The ranking of the baseline system by MLAS is similar (Table 7) but in BLEX, the baseline jumps to rank 13 (Table 8). Besides the simple explanation that UDPipe 1.2 is good at lemmatization, we could also hypothesize that some teams put less effort in building lemmatization models (see also the last column in Table 10).

Each ranking has a different winning system, although the other two winners are typically closely following. The same 8-10 systems occupy best positions in all three tables, though with variable mutual ranking. Some teams seem to have deliberately neglected some of the evaluated attributes: Uppsala is rank 7 in LAS and MLAS, but 24 in

\begin{tabular}{|l|r|}
\hline \multicolumn{1}{|l|}{ Team } & LAS \\
\hline 1. HIT-SCIR (Che et al.) & 75.84 \\
\hline 2. TurkuNLP (Kanerva et al.) & 73.28 \\
\hline 3. UDPipe Future (Straka) & 73.11 \\
LATTICE (Lim et al.) & 73.02 \\
ICS PAS (Rybak and Wróblewska) & 73.02 \\
\hline 6. CEA LIST (Duthoo and Mesnard) & 72.56 \\
\hline 7. Uppsala (Smith et al.) & 72.37 \\
Stanford (Qi et al.) & 72.29 \\
\hline 9. AntNLP (Ji et al.) & 70.90 \\
\hline NLP-Cube (Boros et al.) & 70.82 \\
\hline 11. ParisNLP (Jawahar et al.) & 70.64 \\
\hline 12. SLT-Interactions (Bhat et al.) & 69.98 \\
\hline 13. IBM NY (Wan et al.) & 69.11 \\
\hline 14. UniMelb (Nguyen and Verspoor) & 68.66 \\
\hline 15. LeisureX (Li et al.) & 68.31 \\
\hline 16. KParse (Kurnap et al.) & 66.58 \\
\hline 17. Fudan (Chen et al.) & 66.34 \\
\hline 18. BASELINE UDPipe 1.2 & 65.80 \\
\hline 19. Phoenix (Wu et al.) & 65.61 \\
\hline 20. CUNI x-ling (Rosa and Mareček) & 64.87 \\
\hline 21. BOUN (Özateş et al.) & 63.54 \\
\hline 22. ONLP lab (Seker et al.) & 58.35 \\
\hline 23. iParse (no paper) & 55.83 \\
\hline 24. HUJI (Hershcovich et al.) & 53.69 \\
\hline 25. ArmParser (Arakelyan et al.) & 47.02 \\
\hline 26. SParse (Önder et al.) & 1.95 \\
\hline
\end{tabular}

Table 6: Ranking of the participating systems by the labeled attachment $\mathrm{F}_{1}$-score (LAS), macroaveraged over 82 test sets. Pairs of systems with significantly $(p<0.05)$ different LAS are separated by a line. Citations refer to the corresponding system-description papers in this volume.

BLEX; IBM NY is rank 13 in LAS but 24 in MLAS and 23 in BLEX.

While the LAS scores on individual treebanks are comparable to the 2017 task, the macro average is not, because the set of treebanks is different, and the impact of low-resource languages seems to be higher in the present task.

We used bootstrap resampling to compute $95 \%$ confidence intervals: they are in the range \pm 0.11 to \pm 0.16 (\% LAS/MLAS/BLEX) for all systems except SParse (where it is \pm 0.00 ). 


\begin{tabular}{|l|r|}
\hline \multicolumn{1}{|l|}{ Team } & MLAS \\
\hline 1. UDPipe Future (Praha) & 61.25 \\
\hline 2. TurkuNLP (Turku) & 60.99 \\
Stanford (Stanford) & 60.92 \\
\hline 4. ICS PAS (Warszawa) & 60.25 \\
\hline 5. CEA LIST (Paris) & 59.92 \\
\hline 6. HIT-SCIR (Harbin) & 59.78 \\
\hline 7. Uppsala (Uppsala) & 59.20 \\
\hline 8. NLP-Cube (Bucuresti) & 57.32 \\
\hline 9. LATTICE (Paris) & 57.01 \\
\hline 10. AntNLP (Shanghai) & 55.92 \\
\hline 11. ParisNLP (Paris) & 55.74 \\
\hline 12. SLT-Interactions (Bengaluru) & 54.52 \\
\hline 13. LeisureX (Shanghai) & 53.70 \\
\hline UniMelb (Melbourne) & 53.62 \\
\hline 15. KParse (İstanbul) & 53.25 \\
\hline 16. Fudan (Shanghai) & 52.69 \\
\hline 17. BASELINE UDPipe 1.2 & 52.42 \\
\hline Phoenix (Shanghai) & 52.26 \\
\hline 19. BOUN (İstanbul) & 50.40 \\
CUNI x-ling (Praha) & 50.35 \\
\hline 21. ONLP lab (Ra'anana) & 46.09 \\
\hline 22. iParse (Pittsburgh) & 45.65 \\
\hline 23. HUJI (Yerushalayim) & 44.60 \\
\hline 24. IBM NY (Yorktown Heights) & 40.61 \\
\hline 25. ArmParser (Yerevan) & 36.28 \\
\hline 26. SParse (İstanbul) & 1.68 \\
\hline & \\
\hline
\end{tabular}

Table 7: Ranking of the participating systems by MLAS, macro-averaged over 82 test sets. Pairs of systems with significantly $(p<0.05)$ different MLAS are separated by a line.

We used paired bootstrap resampling to compute whether the difference between two neighboring systems is significant $(p<0.05) .{ }^{10}$

\subsection{Secondary Metrics}

In addition to the main LAS ranking, we evaluated the systems along multiple other axes, which may shed more light on their strengths and weaknesses. This section provides an overview of selected secondary metrics for systems matching or surpassing the baseline; a large number of additional results are available at the shared task website. ${ }^{11}$

The website also features a LAS ranking of unofficial system runs, i.e. those that were not

\footnotetext{
${ }^{10}$ Using Udapi (Popel et al., 2017) eval.Conl118, marked by the presence or absence of horizontal lines in Tables 6-8.

${ }^{11}$ http: // universaldependencies.org/ conll18/results.html
}

\begin{tabular}{|l|r|}
\hline \multicolumn{1}{|l|}{ Team } & BLEX \\
\hline 1. TurkuNLP (Turku) & 66.09 \\
\hline 2. HIT-SCIR (Harbin) & 65.33 \\
\hline 3. UDPipe Future (Praha) & 64.49 \\
ICS PAS (Warszawa) & 64.44 \\
\hline 5. Stanford (Stanford) & 64.04 \\
\hline 6. LATTICE (Paris) & 62.39 \\
CEA LIST (Paris) & 62.23 \\
\hline 8. AntNLP (Shanghai) & 60.91 \\
\hline 9. ParisNLP (Paris) & 60.70 \\
\hline 10. SLT-Interactions (Bengaluru) & 59.68 \\
\hline 11. UniMelb (Melbourne) & 58.67 \\
\hline 12. LeisureX (Shanghai) & 58.42 \\
\hline 13. BASELINE UDPipe 1.2 & 55.80 \\
\hline \multicolumn{1}{|c}{ Phoenix (Shanghai) } & 55.71 \\
\hline 15. NLP-Cube (Bucureşti) & 55.52 \\
\hline 16. KParse (İstanbul) & 55.26 \\
\hline 17. CUNI x-ling (Praha) & 54.07 \\
\hline Fudan (Shanghai) & 54.03 \\
\hline 19. BOUN (İstanbul) & 53.45 \\
\hline 20. iParse (Pittsburgh) & 48.71 \\
\hline 21. HUJI (Yerushalayim) & 48.05 \\
\hline 22. ArmParser (Yerevan) & 39.18 \\
\hline 23. IBM NY (Yorktown Heights) & 32.55 \\
\hline 24. Uppsala (Uppsala) & 32.09 \\
\hline 25. ONLP lab (Ra'anana) & 28.29 \\
\hline 26. SParse (İstanbul) & 1.71 \\
\hline & \\
\hline
\end{tabular}

Table 8: Ranking of the participating systems by BLEX, macro-averaged over 82 test sets. Pairs of systems with significantly $(p<0.05)$ different BLEX are separated by a line.

marked by their teams as primary runs, or were even run after the official evaluation phase closed and test data were unblinded. The difference from the official results is much less dramatic than in 2017, with the exception of the team SParse, who managed to fix their software and produce more valid output files.

As an experiment, we also applied the 2017 system submissions to the 2018 test data. This allows us to test how many systems can actually be used to produce new data without a glitch, as well as to see to what extent the results change over one year and two releases of UD. Here it should be noted that not all of the 2018 task languages and treebanks were present in the 2017 task, therefore causing many systems fail due to an unknown language or treebank code. The full results of this 


\begin{tabular}{|l|r|r|r|}
\hline \multicolumn{1}{|l|}{ Team } & Toks & Wrds & Sents \\
\hline 1. Uppsala & 97.60 & 98.18 & 83.80 \\
\hline 2. HIT-SCIR & $\mathbf{9 8 . 4 2}$ & 98.12 & $\mathbf{8 3 . 8 7}$ \\
\hline 3. CEA LIST & 98.16 & 97.78 & 82.79 \\
\hline 4. CUNI x-ling & 98.09 & 97.74 & $\mathbf{8 2 . 8 0}$ \\
\hline 5. TurkuNLP & 97.83 & 97.42 & $\mathbf{8 3 . 0 3}$ \\
\hline 6. SLT-Interactions & 97.51 & 97.09 & 83.01 \\
\hline 7. UDPipe Future & 97.46 & 97.04 & $\mathbf{8 3 . 6 4}$ \\
\hline 8. Phoenix & 97.46 & 97.03 & 82.91 \\
\hline 9. BASELINE UDPipe & 97.39 & 96.97 & $\mathbf{8 3 . 0 1}$ \\
\hline ParisNLP & 97.39 & 96.97 & 83.01 \\
\hline AntNLP & 97.39 & 96.97 & 83.01 \\
\hline UniMelb & 97.39 & 96.97 & 83.01 \\
\hline BOUN & 97.39 & 96.97 & 83.01 \\
\hline ICS PAS & 97.39 & 96.97 & 83.01 \\
\hline LATTICE & 97.39 & 96.97 & 83.01 \\
\hline LeisureX & 97.39 & 96.97 & 83.01 \\
\hline KParse & 97.39 & 96.97 & 83.01 \\
\hline 18. Fudan & 97.38 & 96.96 & 82.85 \\
\hline 19. IBM NY & 97.30 & 96.92 & $\mathbf{8 3 . 5 1}$ \\
\hline 20. ONLP lab & 97.28 & 96.86 & 83.00 \\
\hline 21. NLP-Cube & $\mathbf{9 7 . 3 6}$ & 96.80 & 82.55 \\
\hline 22. Stanford & 96.19 & 95.99 & 76.55 \\
\hline 23. HUJI & 94.95 & 94.61 & $\mathbf{8 0 . 8 4}$ \\
\hline 24. ArmParser & 79.75 & 79.41 & 13.33 \\
\hline 25. iParse & 78.45 & 78.11 & $\mathbf{6 8 . 3 7}$ \\
\hline 26. SParse & 2.32 & 2.32 & 2.34 \\
\hline & & & \\
\hline
\end{tabular}

Table 9: Tokenization, word segmentation and sentence segmentation (ordered by word $F_{1}$ scores; out-of-order scores in the other two columns are bold).

experiment are available on the shared task website. $^{12}$

Table 9 evaluates detection of tokens, syntactic words and sentences. About a third of the systems trusted the baseline segmentation; this is less than in 2017. For most languages and in aggregate, the segmentation scores are very high and their impact on parsing scores is not easy to prove; but it likely played a role in languages where segmentation is hard. For example, HIT-SCIR's word segmentation in Vietnamese surpasses the second system by a margin of 6 percent points; likewise, the system's advantage in LAS and MLAS (but not in BLEX!) amounts to 7-8 points. Similarly, Uppsala and ParisNLP achieved good segmenta-

\footnotetext{
${ }^{12}$ http: / / universaldependencies.org/ conl118/results-2017-systems.html
}

\begin{tabular}{|l|r|r|r|}
\hline \multicolumn{1}{|c|}{ Team } & UPOS & Feats & Lemm \\
\hline 1. Uppsala & 90.91 & 87.59 & 58.50 \\
\hline 2. HIT-SCIR & 90.19 & 84.24 & $\mathbf{8 8 . 8 2}$ \\
\hline 3. CEA LIST & 89.97 & $\mathbf{8 6 . 8 3}$ & $\mathbf{8 8 . 9 0}$ \\
\hline 4. TurkuNLP & 89.81 & 86.70 & $\mathbf{9 1 . 2 4}$ \\
\hline 5. LATTICE & 89.53 & 83.74 & 87.84 \\
\hline 6. UDPipe Future & 89.37 & $\mathbf{8 6 . 6 7}$ & $\mathbf{8 9 . 3 2}$ \\
\hline 7. Stanford & 89.01 & 85.47 & 88.32 \\
\hline 8. ICS PAS & 88.70 & 85.14 & 87.99 \\
\hline 9. CUNI x-ling & 88.68 & 84.56 & $\mathbf{8 8 . 9 6}$ \\
\hline 10. NLP-Cube & 88.50 & $\mathbf{8 5 . 0 8}$ & 81.21 \\
\hline 11. SLT-Interactions & 88.12 & 83.72 & $\mathbf{8 7 . 5 1}$ \\
\hline 12. IBM NY & 88.02 & 59.11 & 59.51 \\
\hline 13. UniMelb & 87.90 & $\mathbf{8 3 . 7 4}$ & $\mathbf{8 7 . 8 4}$ \\
\hline 14. KParse & 87.62 & $\mathbf{8 4 . 3 2}$ & 86.26 \\
\hline 15. Phoenix & 87.49 & 83.87 & $\mathbf{8 7 . 6 9}$ \\
\hline 16. ParisNLP & 87.35 & 83.74 & $\mathbf{8 7 . 8 4}$ \\
\hline 17. BASELINE UDPipe & 87.32 & 83.74 & 87.84 \\
\hline AntNLP & 87.32 & 83.74 & 87.84 \\
\hline 19. ONLP lab & 87.25 & 83.67 & 57.10 \\
\hline 20. Fudan & 87.25 & 83.47 & $\mathbf{8 5 . 9 1}$ \\
\hline 21. BOUN & 87.19 & $\mathbf{8 3 . 7 3}$ & $\mathbf{8 7 . 6 8}$ \\
\hline 22. LeisureX & 87.15 & 83.46 & $\mathbf{8 7 . 7 7}$ \\
\hline 23. HUJI & 85.06 & 81.51 & 85.61 \\
\hline 24. ArmParser & 72.99 & 69.91 & 72.22 \\
\hline 25. iParse & 71.38 & 68.64 & 71.68 \\
\hline 26. SParse & 2.25 & 2.29 & 2.28 \\
\hline
\end{tabular}

Table 10: Universal POS tags, features and lemmas (ordered by UPOS $F_{1}$ scores; out-of-order scores in the other two columns are bold).

tion scores (better than their respective macroaverages) on Arabic. They were able to translate it into better LAS, but not MLAS and BLEX, where there were too many other chances to make an error.

The complexity of the new metrics, especially MLAS, is further underlined by Table 10: Uppsala is the clear winner in both UPOS tags and morphological features, but 6 other teams had better dependency relations and better MLAS. Note that as with segmentation, morphology predicted by the baseline system was available, though only a few systems seem to have used it without attempting to improve it.

\subsection{Partial Results}

Table 11 gives the three main scores averaged over the 61 "big" treebanks (training data larger than 


\begin{tabular}{|l|r|r|r|}
\hline \multicolumn{1}{|l|}{ Team } & LAS & MLAS & BLEX \\
\hline 1. HIT-SCIR & 84.37 & 70.12 & 75.05 \\
\hline 2. Stanford & 83.03 & $\mathbf{7 2 . 6 7}$ & $\mathbf{7 5 . 4 6}$ \\
\hline 3. TurkuNLP & 81.85 & 71.27 & $\mathbf{7 5 . 8 3}$ \\
\hline 4. UDPipe Future & 81.83 & $\mathbf{7 1 . 7 1}$ & 74.67 \\
\hline 5. ICS PAS & 81.72 & 70.30 & 74.42 \\
\hline 6. CEA LIST & 81.66 & $\mathbf{7 0 . 8 9}$ & 72.32 \\
\hline 7. LATTICE & 80.97 & 66.27 & 71.50 \\
\hline 8. NLP-Cube & 80.48 & $\mathbf{6 7 . 7 9}$ & 64.76 \\
\hline 9. ParisNLP & 80.29 & 65.88 & $\mathbf{7 0 . 9 5}$ \\
\hline 10. Uppsala & 80.25 & $\mathbf{6 8 . 8 1}$ & 36.02 \\
\hline 11. SLT-Interactions & 79.67 & 64.95 & $\mathbf{6 9 . 7 7}$ \\
\hline 12. AntNLP & 79.61 & $\mathbf{6 5 . 4 3}$ & $\mathbf{7 0 . 3 4}$ \\
\hline 13. LeisureX & 77.98 & 63.79 & 68.55 \\
\hline 14. UniMelb & 77.69 & 63.17 & 68.25 \\
\hline 15. IBM NY & 77.55 & 47.34 & 36.68 \\
\hline 16. Fudan & 75.42 & $\mathbf{6 2 . 2 8}$ & $\mathbf{6 2 . 9 0}$ \\
\hline 17. KParse & 74.84 & $\mathbf{6 2 . 4 0}$ & $\mathbf{6 3 . 8 4}$ \\
\hline 18. BASELINE UDPipe & 74.14 & 61.27 & $\mathbf{6 4 . 6 7}$ \\
\hline 19. Phoenix & 73.93 & 61.12 & 64.47 \\
\hline 20. BOUN & 72.85 & 60.00 & 62.99 \\
\hline 21. CUNI x-ling & 71.54 & 58.33 & 61.63 \\
\hline 22. ONLP lab & 67.08 & 55.20 & 33.08 \\
\hline 23. iParse & 66.55 & $\mathbf{5 5 . 3 7}$ & $\mathbf{5 8 . 8 0}$ \\
\hline 24. HUJI & 62.07 & 53.20 & 56.90 \\
\hline 25. ArmParser & 58.14 & 45.87 & 49.25 \\
\hline 26. SParse & 2.63 & 2.26 & 2.30 \\
\hline & & & \\
\hline
\end{tabular}

\begin{tabular}{|l|r|r|r|}
\hline \multicolumn{1}{|c|}{ Team } & LAS & MLAS & BLEX \\
\hline 1. CUNI x-ling & 27.89 & 6.13 & 13.98 \\
\hline 2. Uppsala & 25.87 & 5.16 & 9.03 \\
\hline 3. CEA LIST & 23.90 & 3.75 & $\mathbf{1 0 . 9 9}$ \\
\hline 4. HIT-SCIR & 23.88 & 2.88 & 10.50 \\
\hline 5. LATTICE & 23.39 & $\mathbf{4 . 3 8}$ & 10.01 \\
\hline 6. TurkuNLP & 22.91 & 3.59 & $\mathbf{1 1 . 4 0}$ \\
\hline 7. IBM NY & 21.88 & 2.62 & 7.17 \\
\hline 8. UDPipe Future & 21.75 & $\mathbf{2 . 8 2}$ & $\mathbf{8 . 8 0}$ \\
\hline 9. ICS PAS & 19.26 & 1.89 & 6.17 \\
\hline 10. AntNLP & 18.59 & $\mathbf{3 . 4 3}$ & $\mathbf{8 . 6 1}$ \\
\hline 11. KParse & 17.84 & 3.32 & 6.58 \\
\hline 12. SLT-Interactions & 17.47 & 1.79 & $\mathbf{6 . 9 5}$ \\
\hline 13. Stanford & 17.45 & $\mathbf{2 . 7 6}$ & $\mathbf{7 . 6 3}$ \\
\hline 14. BASELINE UDPipe & 17.17 & $\mathbf{3 . 4 4}$ & 7.63 \\
\hline UniMelb & 17.17 & 3.44 & 7.63 \\
\hline 16. LeisureX & 17.16 & 3.43 & 7.63 \\
\hline 17. Phoenix & 16.99 & 3.02 & $\mathbf{8 . 0 0}$ \\
\hline 18. NLP-Cube & 16.85 & $\mathbf{3 . 3 9}$ & 7.05 \\
\hline 19. ParisNLP & 16.52 & 2.53 & 6.75 \\
\hline 20. ONLP lab & 15.98 & $\mathbf{3 . 5 8}$ & 4.96 \\
\hline 21. Fudan & 15.45 & 2.98 & $\mathbf{6 . 6 1}$ \\
\hline 22. BOUN & 14.78 & 2.59 & 6.43 \\
\hline 23. HUJI & 8.53 & 0.92 & 2.77 \\
\hline 24. ArmParser & 7.47 & $\mathbf{1 . 8 6}$ & $\mathbf{3 . 5 4}$ \\
\hline 25. iParse & 2.82 & 0.23 & 0.97 \\
\hline 26. SParse & 0.00 & 0.00 & 0.00 \\
\hline
\end{tabular}

Table 11: Average LAS on the 61 "big" treebanks (ordered by LAS $\mathrm{F}_{1}$ scores; out-of-order scores in the other two columns are bold).

test data, development data available). Higher scores reflect the fact that models for these test sets are easier to learn: enough data is available, no cross-lingual or cross-domain learning is necessary (the extra test sets are not included here). Regarding ranking, the Stanford system makes a remarkable jump when it does not have to carry the load of underresourced languages: from rank 8 to 2 in LAS, from 3 to 1 in MLAS and from 5 to 2 in BLEX.

Table 12 gives the LAS $F_{1}$ score on the nine low-resource languages only. Here we have a true specialist: The team CUNI $x$-ling lives up to its name and wins in all three scores, although in the overall ranking they fall even slightly behind the baseline. On the other hand, the scores are extremely low and the outputs are hardly useful for any downstream application. Especially morphol-

ogy is almost impossible to learn from foreign languages, hence the much lower values of MLAS and BLEX. BLEX is a bit better than MLAS, which could be explained by cases where a word form is identical to its lemma. However, there are significant language-by-language differences; the best LAS on Faroese and Upper Sorbian surpassing $45 \%$. This probably owes to the presence of many Germanic and Slavic treebanks in training data, including some of the largest datasets in UD. Three languages, Buryat, Kurmanji and Upper Sorbian, were introduced in the 2017 task as 


\begin{tabular}{|l|r|r|r|}
\hline Team & LAS & MLAS & BLEX \\
\hline 1. HIT-SCIR & 69.53 & 45.94 & 53.30 \\
\hline 2. LATTICE & 68.12 & 45.03 & 51.71 \\
\hline 3. ICS PAS & 66.90 & $\mathbf{4 9 . 2 4}$ & $\mathbf{5 4 . 8 9}$ \\
\hline 4. TurkuNLP & 64.48 & 47.63 & 53.54 \\
\hline 5. UDPipe Future & 64.21 & 47.53 & 49.53 \\
\hline 6. AntNLP & 63.73 & 42.24 & 48.31 \\
\hline 7. Uppsala & 63.60 & $\mathbf{4 6 . 0 0}$ & 29.25 \\
\hline 8. ParisNLP & 60.84 & 40.71 & $\mathbf{4 6 . 0 8}$ \\
\hline 9. CEA LIST & 57.34 & 39.97 & 43.39 \\
\hline 10. KParse & 57.32 & 39.20 & $\mathbf{4 3 . 6 1}$ \\
\hline 11. NLP-Cube & 56.78 & 37.13 & 38.30 \\
\hline 12. SLT-Interactions & 56.74 & 35.73 & $\mathbf{4 2 . 9 0}$ \\
\hline 13. IBM NY & 56.13 & 26.51 & 25.23 \\
\hline 14. UniMelb & 56.12 & $\mathbf{3 6 . 0 9}$ & $\mathbf{4 2 . 0 9}$ \\
\hline 15. BASELINE UDPipe & 55.01 & $\mathbf{3 8 . 8 0}$ & 41.06 \\
\hline LeisureX & 55.01 & 38.80 & 41.06 \\
\hline 17. Phoenix & 54.63 & 38.38 & 40.72 \\
\hline Fudan & 54.63 & 38.15 & 40.07 \\
\hline 19. CUNI x-ling & 54.33 & 38.10 & $\mathbf{4 0 . 7 0}$ \\
\hline 20. BOUN & 50.18 & 34.29 & 36.75 \\
\hline 21. Stanford & 48.56 & $\mathbf{3 4 . 8 6}$ & $\mathbf{3 8 . 5 5}$ \\
\hline 22. ONLP lab & 47.49 & 32.74 & 22.39 \\
\hline 23. iParse & 38.79 & 28.03 & $\mathbf{2 9 . 6 2}$ \\
\hline 24. HUJI & 36.74 & 24.47 & 27.70 \\
\hline 25. ArmParser & 34.54 & 22.94 & 25.26 \\
\hline 26. SParse & 0.00 & 0.00 & 0.00 \\
\hline & & & \\
\hline
\end{tabular}

Table 13: Average attachment score on the 7 small treebanks: Galician TreeGal, Irish, Latin Perseus, North Sámi, Norwegian Nynorsk LIA, Russian Taiga and Slovenian SST (ordered by LAS $F_{1}$ scores; out-of-order scores in the other two columns are bold).

surprise languages and had higher scores there. ${ }^{13}$ This is because in 2017, the segmentation, POS tags and morphology UDPipe models were trained on the test data, applied to it via cross-validation, and made available to the systems. Such an approach makes the conditions unrealistic, therefore it was not repeated this year. Consequently, parsing these languages is now much harder.

In contrast, the results on the 7 treebanks with "small" training data and no development data (Table 13) are higher on average, but again the variance is significant. The smallest treebank

\footnotetext{
${ }^{13}$ The fourth surprise language, North Sámi, has now additional training data and does not fall in the low-resource category.
}

\begin{tabular}{|l|r|r|r|}
\hline \multicolumn{1}{|c|}{ Team } & LAS & MLAS & BLEX \\
\hline 1. HIT-SCIR & 74.20 & 55.52 & 62.34 \\
\hline 2. Stanford & 73.14 & $\mathbf{5 8 . 7 5}$ & 61.96 \\
\hline 3. LATTICE & 72.34 & 55.60 & 60.42 \\
\hline 4. Uppsala & 72.27 & $\mathbf{5 7 . 8 0}$ & 29.73 \\
\hline 5. ICS PAS & 72.18 & $\mathbf{5 8 . 0 7}$ & $\mathbf{6 0 . 9 7}$ \\
\hline 6. TurkuNLP & 71.78 & 57.54 & $\mathbf{6 3 . 2 5}$ \\
\hline 7. UDPipe Future & 71.57 & $\mathbf{5 7 . 9 3}$ & 61.52 \\
\hline 8. CEA LIST & 70.45 & 54.99 & 57.83 \\
\hline 9. NLP-Cube & 69.83 & $\mathbf{5 5 . 0 1}$ & 54.15 \\
\hline 10. IBM NY & 69.40 & 46.59 & 38.12 \\
\hline 11. AntNLP & 68.87 & $\mathbf{5 3 . 4 7}$ & $\mathbf{5 7 . 7 1}$ \\
\hline 12. UniMelb & 68.72 & 52.05 & 56.77 \\
\hline 13. Phoenix & 66.97 & $\mathbf{5 2 . 2 6}$ & 55.69 \\
\hline 14. BASELINE UDPipe & 66.63 & 51.75 & 54.87 \\
\hline 15. KParse & 66.55 & 51.29 & 54.45 \\
\hline 16. SLT-Interactions & 64.73 & 48.47 & $\mathbf{5 4 . 9 0}$ \\
\hline 17. CUNI x-ling & 64.70 & $\mathbf{4 9 . 7 1}$ & 52.72 \\
\hline 18. ParisNLP & 64.09 & 48.79 & $\mathbf{5 3 . 1 6}$ \\
\hline 19. Fudan & 63.54 & 45.54 & 50.73 \\
\hline 20. LeisureX & 61.05 & 41.95 & 50.60 \\
\hline 21. BOUN & 56.46 & 41.91 & 45.12 \\
\hline 22. HUJI & 56.35 & $\mathbf{4 6 . 5 2}$ & $\mathbf{5 0 . 1 0}$ \\
\hline 23. iParse & 44.20 & 33.43 & 38.18 \\
\hline 24. ONLP lab & 43.33 & 30.20 & 20.08 \\
\hline 25. ArmParser & 0.00 & 0.00 & 0.00 \\
\hline SParse & 0.00 & 0.00 & 0.00 \\
\hline
\end{tabular}

Table 14: Average attachment score on the 5 additional test sets for high-resource languages: Czech PUD, English PUD, Finnish PUD, Japanese Modern and Swedish PUD (ordered by LAS $F_{1}$ scores; out-of-order scores in the other two columns are bold).

in the group, Norwegian Nynorsk LIA, has only 3583 training words. There are two larger Norwegian treebanks that could be used as additional training sources. However, the LIA treebank consists of spoken dialects and is probably quite dissimilar to the other treebanks. The same can be said about Slovenian SST and the other Slovenian treebank; SST is the most difficult dataset in the group, despite of having almost $20 \mathrm{~K}$ of its own training words. Other treebanks, like Russian Taiga and Galician TreeGal, have much better scores (74\% LAS, about 61\% MLAS and 64\% BLEX). There are also two treebanks that are the sole representatives of their languages: Irish and North Sámi. Their best LAS is around 70\%: com- 
parable to Nynorsk LIA but much better than SST. ICS PAS is the most successful system in the domain of small treebanks, especially when judged by MLAS and BLEX.

Table 14 gives the average LAS on the 5 extra test sets (no own training data, but other treebanks of the same language exist). Four of them come from the Parallel UD (PUD) collection introduced in the 2017 task (Zeman et al., 2017). The fifth, Japanese Modern, turned out to be one of the toughest test sets in this shared task. There is another Japanese treebank, GSD, with over 160K training tokens, but the Modern dataset seems almost inapproachable with models trained on GSD. A closer inspection reveals why: despite its name, it is actually a corpus of historical Japanese, although from the relatively recent Meiji and Taishō periods (1868-1926). An average sentence in GSD is about $1.3 \times$ longer than in Modern. GSD has significantly more tokens tagged as auxiliaries, but more importantly, the top ten AUX lemmas in the two treebanks are completely disjoint sets. Some other words are out-of-vocabulary because their preferred spelling changed. For instance, the demonstrative pronoun sore is written using hiragana in GSD, but a kanji character is used in Modern. Striking differences can be observed also in dependency relations: in GSD, $3.7 \%$ relations are nsubj (subject), and $1.2 \%$ are cop (copula). In Modern, there is just $0.13 \%$ of subjects, and not a single occurrence of a copula.

See Tables 15, 16 and 17 for a ranking of all test sets by the best scores achieved on them by any parser. Note that this cannot be directly interpreted as a ranking of languages by their parsing difficulty: many treebanks have high ranks simply because the corresponding training data is large. Table 18 compares average LAS and MLAS for each treebank.

Finally, Tables 19 and 20 show the treebanks where word and sentence segmentation was extremely difficult (judged by the average parser score). Not surprisingly, word segmentation is difficult for the low-resource languages and for languages like Chinese, Vietnamese, Japanese and Thai, where spaces do not separate words. Notably the Japanese GSD set is not as difficult, but whoever trusted it, crashed on the "Modern" set. Sentence segmentation was particularly hard for treebanks without punctuation, i.e., most of the classical languages and spoken data.

\begin{tabular}{|c|c|c|c|c|}
\hline Treebank & LAS & Best system & Avg & StDev \\
\hline 1. pl_lfg & 94.86 & HIT-SCIR & $\begin{array}{l}85.89 \\
\end{array}$ & \pm 6.97 \\
\hline 2. ru_syntagrus & 92.48 & HIT-SCIR & 79.68 & \pm 9.09 \\
\hline 3. hi_hdtb & 92.41 & HIT-SCIR & 85.16 & \pm 5.32 \\
\hline 4. $\mathrm{pl} \_\mathrm{sz}$ & 92.23 & HIT-SCIR & 81.47 & \pm 7.27 \\
\hline 5. cs_fictree & 92.02 & HIT-SCIR & 82.10 & \pm 7.26 \\
\hline 6. it_isdt & 92.00 & HIT-SCIR & 87.61 & \pm 4.12 \\
\hline 7. cs_pdt & 91.68 & HIT-SCIR & 82.18 & \pm 6.91 \\
\hline 8. ca_ancora & 91.61 & HIT-SCIR & 83.61 & \pm 6.01 \\
\hline 9. cs_cac & 91.61 & HIT-SCIR & 82.69 & \pm 6.93 \\
\hline 10. sl_ssj & 91.47 & HIT-SCIR & 75.00 & \pm 9.13 \\
\hline 11. no_bokmaal & 91.23 & HIT-SCIR & 79.80 & \pm 7.29 \\
\hline 12. bg_btb & 91.22 & HIT-SCIR & 82.52 & \pm 5.88 \\
\hline 13. no_nynorsk & 90.99 & HIT-SCIR & 78.55 & \pm 7.88 \\
\hline 14. es_ancora & 90.93 & HIT-SCIR & 82.84 & \pm 6.17 \\
\hline 15. fi_pud & 90.23 & HIT-SCIR & 68.87 & \pm 15.61 \\
\hline 16. fr_sequoia & 89.89 & LATTICE & 80.55 & \pm 5.91 \\
\hline 17. el_gdt & 89.65 & HIT-SCIR & 80.65 & \pm 6.05 \\
\hline 18. nl_alpino & 89.56 & HIT-SCIR & 77.76 & \pm 7.42 \\
\hline 19. sk_snk & 88.85 & HIT-SCIR & 76.53 & \pm 7.24 \\
\hline 20. fi_tdt & 88.73 & HIT-SCIR & 73.55 & \pm 9.39 \\
\hline 21. sr_set & 88.66 & Stanford & 79.84 & \pm 6.57 \\
\hline 22. sv_talbanken & 88.63 & HIT-SCIR & 77.71 & \pm 6.50 \\
\hline 23. fi_ftb & 88.53 & HIT-SCIR & 76.89 & \pm 7.60 \\
\hline 24. uk_iu & 88.43 & HIT-SCIR & 72.47 & \pm 8.25 \\
\hline 25. fa_seraji & 88.11 & HIT-SCIR & 78.71 & \pm 6.04 \\
\hline 26. en_pud & 87.89 & LATTICE & 74.51 & \pm 8.28 \\
\hline 27. pt_bosque & 87.81 & Stanford & 80.49 & \pm 5.46 \\
\hline 28. hr_set & 87.36 & HIT-SCIR & 78.37 & \pm 6.42 \\
\hline 29. fro_sremf & 87.12 & UDPipe Future & 74.38 & \pm 16.74 \\
\hline 30. la_ittb & 87.08 & HIT-SCIR & 77.00 & \pm 7.42 \\
\hline 31. ko_kaist & 86.91 & HIT-SCIR & 77.10 & \pm 8.72 \\
\hline 32. fr_gsd & 86.89 & HIT-SCIR & 79.43 & \pm 5.47 \\
\hline 33. ro_rrt & 86.87 & HIT-SCIR & 75.77 & \pm 7.66 \\
\hline 34. nl_lassysmall & 86.84 & HIT-SCIR & 75.08 & \pm 6.59 \\
\hline 35. da_ddt & 86.28 & HIT-SCIR & 75.02 & \pm 6.47 \\
\hline 36. cs_pud & 86.13 & HIT-SCIR & 73.24 & \pm 9.97 \\
\hline 37. af_afribooms & 85.47 & HIT-SCIR & 76.61 & \pm 6.17 \\
\hline 38. et_edt & 85.35 & HIT-SCIR & 72.08 & \pm 8.71 \\
\hline 39. ko_gsd & 85.14 & HIT-SCIR & 71.88 & \pm 10.53 \\
\hline 40. en_gum & 85.05 & LATTICE & 74.20 & \pm 6.27 \\
\hline 41. en_ewt & 84.57 & HIT-SCIR & 75.99 & \pm 5.40 \\
\hline 42. eu_bdt & 84.22 & HIT-SCIR & 72.08 & \pm 8.83 \\
\hline 43. sv_lines & 84.08 & HIT-SCIR & 73.76 & \pm 5.98 \\
\hline 44. lv_lvtb & 83.97 & HIT-SCIR & 67.76 & \pm 9.01 \\
\hline 45. ur_udtb & 83.39 & HIT-SCIR & 75.89 & \pm 4.69 \\
\hline 46. ja_gsd & 83.11 & HIT-SCIR & 73.68 & \pm 4.55 \\
\hline 47. gl_ctg & 82.76 & Stanford & 72.46 & \pm 7.13 \\
\hline 48. hu_szeged & 82.66 & HIT-SCIR & 67.05 & \pm 8.63 \\
\hline 49. en_lines & 81.97 & HIT-SCIR & 72.28 & \pm 5.59 \\
\hline 50. de_gsd & 80.36 & HIT-SCIR & 70.13 & \pm 7.14 \\
\hline 51. sv_pud & 80.35 & HIT-SCIR & 67.02 & \pm 9.23 \\
\hline 52. id_gsd & 80.05 & HIT-SCIR & 73.05 & \pm 4.69 \\
\hline 53. it_postwita & 79.39 & HIT-SCIR & 64.95 & \pm 6.88 \\
\hline 54. grc_perseus & 79.39 & HIT-SCIR & 59.01 & \pm 15.56 \\
\hline 55. grc_proiel & 79.25 & HIT-SCIR & 65.02 & \pm 14.58 \\
\hline 56. ar_padt & 77.06 & Stanford & 64.07 & \pm 6.41 \\
\hline 57. zh_gsd & 76.77 & HIT-SCIR & 60.32 & \pm 6.14 \\
\hline 58. he_htb & 76.09 & Stanford & 58.73 & \pm 5.29 \\
\hline 59. fr_spoken & 75.78 & HIT-SCIR & 64.66 & \pm 5.35 \\
\hline 60. cu_proiel & 75.73 & Stanford & 62.64 & \pm 6.98 \\
\hline 61. gl_treegal & 74.25 & UDPipe Future & 64.65 & \pm 5.61 \\
\hline 62. ru_taiga & 74.24 & ICS PAS & 56.27 & \pm 9.16 \\
\hline 63. la_proiel & 73.61 & HIT-SCIR & 61.25 & \pm 6.87 \\
\hline 64. la_perseus & 72.63 & HIT-SCIR & 46.91 & \pm 11.12 \\
\hline 65. ga_idt & 70.88 & TurkuNLP & 58.37 & \pm 7.05 \\
\hline 66. no_nynorsklia & 70.34 & HIT-SCIR & 50.33 & \pm 9.28 \\
\hline 67. sme_giella & 69.87 & LATTICE & 51.10 & \pm 14.32 \\
\hline 68. got_proiel & 69.55 & Stanford & 60.55 & \pm 4.93 \\
\hline 69. ug_udt & 67.05 & HIT-SCIR & 54.27 & \pm 6.90 \\
\hline 70. tr_imst & 66.44 & HIT-SCIR & 55.61 & \pm 6.49 \\
\hline 71. sl_sst & 61.39 & HIT-SCIR & 47.07 & \pm 5.84 \\
\hline 72. vi_vtb & 55.22 & HIT-SCIR & 40.40 & \pm 4.43 \\
\hline 73. fo_oft & 49.43 & CUNI $x$-lin & 27.87 & \pm 9.75 \\
\hline 74. hsb_ufal & 46.42 & SLT-Interactions & 26.48 & \pm 8.90 \\
\hline 75. br_keb & 38.64 & CEA LIST & 13.27 & \pm 8.77 \\
\hline 76. hy_armtdp & 37.01 & LATTICE & 22.39 & \pm 7.91 \\
\hline 77. kk_ktb & 31.93 & Uppsala & 19.11 & \pm 6.34 \\
\hline 78. $\mathrm{kmr} \_\mathrm{mg}$ & 30.41 & IBM NY & 20.27 & \pm 6.14 \\
\hline 79. pem_nsc & 30.07 & CUNI $x$-ling & 13.19 & \pm 5.76 \\
\hline 80. ja_modern & 28.33 & Stanford & 18.92 & \pm 5.14 \\
\hline 81. bxr_bdt & 19.53 & AntNLP & 11.45 & \pm 4.28 \\
\hline 82. th_pud & 13.70 & CUNI $x$-ling & 1.38 & \pm 2.83 \\
\hline
\end{tabular}

Table 15: Treebank ranking by best parser LAS (Avg=average LAS over all systems, out-of-order scores in bold). 


\begin{tabular}{|c|c|c|c|c|}
\hline Treebank & MLAS & Best system & Avg & StDev \\
\hline 1. pl_lfg & 86.93 & UDPipe Future & 73.73 & \pm 7.29 \\
\hline 2. ru_syntagrus & 86.76 & UDPipe Future & 71.63 & \pm 9.36 \\
\hline 3. cs_pdt & 85.10 & UDPipe Future & 73.61 & \pm 6.32 \\
\hline 4. cs_fictree & 84.23 & ICS PAS & 69.91 & \pm 7.77 \\
\hline 5. ca_ancora & 84.07 & UDPipe Future & 74.62 & \pm 7.69 \\
\hline 6. es_ancora & 83.93 & Stanford & 74.61 & \pm 7.43 \\
\hline 7. it_isdt & 83.89 & Stanford & 77.14 & \pm 8.89 \\
\hline 8. fi_pud & 83.78 & Stanford & 62.38 & \pm 14.83 \\
\hline 9. no_bokmaal & 83.68 & UDPipe Future & 70.75 & \pm 8.92 \\
\hline 10. cs_cac & 83.42 & UDPipe Future & 71.39 & \pm 6.89 \\
\hline 11. bg_btb & 83.12 & UDPipe Future & 73.18 & \pm 7.15 \\
\hline 12. fr_sequoia & 82.55 & Stanford & 70.42 & \pm 9.04 \\
\hline 13. sl_ssj & 82.38 & Stanford & 62.41 & \pm 9.18 \\
\hline 14. no_nynorsk & 81.86 & UDPipe Future & 68.62 & \pm 9.45 \\
\hline 15. ko_kaist & 81.29 & HIT-SCIR & 70.18 & \pm 9.36 \\
\hline 16. ko_gsd & 80.85 & HIT-SCIR & 63.73 & \pm 16.02 \\
\hline 17. fi_tdt & 80.84 & Stanford & 65.27 & \pm 9.22 \\
\hline 18. fa_seraji & 80.83 & UDPipe Future & 71.23 & \pm 7.77 \\
\hline 19. pl_sz & 80.77 & Stanford & 64.80 & \pm 8.49 \\
\hline 20. fro_sremf & 80.28 & UDPipe Future & 65.19 & \pm 16.58 \\
\hline 21. la_ittb & 79.84 & ICS PAS & 67.77 & \pm 8.37 \\
\hline 22. fifftb & 79.65 & TurkuNLP & 66.11 & \pm 8.86 \\
\hline 23. sv_talbanken & 79.32 & Stanford & 68.05 & \pm 8.49 \\
\hline 24. ro_rrt & 78.68 & TurkuNLP & 67.43 & \pm 7.24 \\
\hline 25. el_gdt & 78.66 & Stanford & 64.29 & \pm 8.28 \\
\hline 26. fr_gsd & 78.44 & Stanford & 69.33 & \pm 8.59 \\
\hline 27. hi_hdtb & 78.30 & UDPipe Future & 68.48 & \pm 5.88 \\
\hline 28. sr_set & 77.73 & UDPipe Future & 67.33 & \pm 5.96 \\
\hline 29. da_ddt & 77.31 & Stanford & 65.00 & \pm 6.89 \\
\hline 30. et_edt & 76.97 & TurkuNLP & 63.59 & \pm 8.34 \\
\hline 31. nl_alpino & 76.52 & Stanford & 62.82 & \pm 9.81 \\
\hline 32. en_ewt & 76.33 & Stanford & 66.84 & \pm 5.86 \\
\hline 33. pt_bosque & 75.94 & Stanford & 66.22 & \pm 6.76 \\
\hline 34. cs_pud & 75.81 & UDPipe Future & 60.47 & \pm 11.36 \\
\hline 35. af_afribooms & 75.67 & UDPipe Future & 63.76 & \pm 7.06 \\
\hline 36. sk_snk & 75.01 & Stanford & 56.82 & \pm 8.32 \\
\hline 37. en_pud & 74.86 & Stanford & 63.05 & \pm 7.89 \\
\hline 38. nl_lassysmall & 74.11 & Stanford & 61.95 & \pm 9.12 \\
\hline 39. hr_set & 73.44 & Stanford & 60.08 & \pm 7.07 \\
\hline 40. en_gum & 73.24 & ICS PAS & 61.72 & \pm 7.69 \\
\hline 41. ja_gsd & 72.62 & HIT-SCIR & 59.52 & \pm 6.20 \\
\hline 42. uk_iu & 72.27 & UDPipe Future & 55.45 & \pm 8.08 \\
\hline 43. en_lines & 72.25 & ICS PAS & 62.35 & \pm 8.04 \\
\hline 44. eu_bdt & 71.73 & UDPipe Future & 58.49 & \pm 8.62 \\
\hline 45. gl_ctg & 70.92 & Stanford & 57.92 & \pm 14.10 \\
\hline 46. ar_padt & 68.54 & Stanford & 53.28 & \pm 6.12 \\
\hline 47. it_postwita & 68.50 & Stanford & 51.72 & \pm 8.80 \\
\hline 48. id_gsd & 68.36 & Stanford & 61.03 & \pm 6.49 \\
\hline 49. lv_lvtb & 67.89 & Stanford & 53.31 & \pm 7.96 \\
\hline 50. hu_szeged & 67.13 & UDPipe Future & 53.08 & \pm 8.01 \\
\hline 51. zh_gsd & 66.62 & HIT-SCIR & 50.42 & \pm 5.87 \\
\hline 52. sv_lines & 66.58 & Stanford & 57.40 & \pm 7.43 \\
\hline 53. fr_spoken & 64.67 & HIT-SCIR & 53.17 & \pm 5.61 \\
\hline 54. he_htb & 63.38 & Stanford & 45.22 & \pm 4.94 \\
\hline 55. cu_proiel & 63.31 & Stanford & 50.28 & \pm 6.69 \\
\hline 56. ru_taiga & 61.59 & ICS PAS & 37.16 & \pm 7.53 \\
\hline 57. gl_treegal & 60.63 & UDPipe Future & 47.35 & \pm 5.93 \\
\hline 58. grc_proiel & 60.27 & Stanford & 47.62 & \pm 11.82 \\
\hline 59. la_proiel & 59.36 & Stanford & 47.79 & \pm 6.90 \\
\hline 60. de_gsd & 58.04 & TurkuNLP & 39.13 & \pm 10.35 \\
\hline 61. ur_udtb & 57.98 & TurkuNLP & 49.64 & \pm 4.21 \\
\hline 62. no_nynorsklia & 57.51 & ICS PAS & 37.08 & \pm 7.78 \\
\hline 63. sme_giella & 57.47 & TurkuNLP & 38.29 & \pm 12.37 \\
\hline 64. got_proiel & 56.45 & UDPipe Future & 46.18 & \pm 5.36 \\
\hline 65. tr_imst & 55.73 & Stanford & 45.26 & \pm 6.15 \\
\hline 66. grc_perseus & 54.98 & HIT-SCIR & 35.65 & \pm 12.31 \\
\hline 67. sv_pud & 51.74 & TurkuNLP & 39.41 & \pm 7.78 \\
\hline 68. la_perseus & 49.77 & ICS PAS & 28.67 & \pm 8.06 \\
\hline 69. vi_vtb & 47.61 & HIT-SCIR & 32.45 & \pm 7.28 \\
\hline 70. sl_sst & 45.93 & ICS PAS & 33.12 & \pm 5.33 \\
\hline 71. ga_idt & 45.79 & TurkuNLP & 33.70 & \pm 5.18 \\
\hline 72. ug_udt & 45.78 & UDPipe Future & 35.08 & \pm 5.96 \\
\hline 73. br_keb & 13.91 & Uppsala & 1.52 & \pm 3.34 \\
\hline 74. hy_armtdp & 13.36 & CUNI $x$-ling & 5.94 & \pm 2.92 \\
\hline 75. ja_modern & 11.82 & Uppsala & 6.45 & \pm 2.59 \\
\hline 76. hsb_ufal & 9.09 & LATTICE & 4.66 & \pm 2.37 \\
\hline 77. kk_ktb & 8.93 & CUNI $x$-ling & 5.04 & \pm 2.34 \\
\hline 78. $\mathrm{kmr} \_\mathrm{mg}$ & 7.98 & IBM NY & 4.01 & \pm 1.96 \\
\hline 79. th_pud & 6.29 & CUNI x-ling & 0.42 & \pm 1.27 \\
\hline 80. pcm_nsc & 5.30 & KParse & 3.00 & \pm 1.30 \\
\hline 81. bxr_bdt & 2.98 & AntNLP & 1.33 & \pm 0.72 \\
\hline 82. fo_oft & 1.07 & CUNI $x$-ling & 0.37 & \pm 0.21 \\
\hline
\end{tabular}

\begin{tabular}{|c|c|c|c|c|}
\hline Treebank & BLEX & Best system & Avg & StDev \\
\hline 1. pl_lfg & 90.42 & TurkuNLP & 72.81 & \pm 16.96 \\
\hline 2. ru_syntagrus & 88.65 & TurkuNLP & 68.57 & \pm 18.07 \\
\hline 3. cs_pdt & 87.91 & HIT-SCIR & 74.41 & \pm 14.88 \\
\hline 4. cs_fictree & 87.81 & ICS PAS & 71.10 & \pm 16.26 \\
\hline 5. cs_cac & 86.79 & TurkuNLP & 71.61 & \pm 18.18 \\
\hline 6. hi_hdtb & 86.74 & HIT-SCIR & 75.80 & \pm 9.28 \\
\hline 7. pl_sz & 86.29 & TurkuNLP & 67.33 & \pm 17.15 \\
\hline 8. no_bokmaal & 85.82 & UDPipe Future & 69.52 & \pm 13.54 \\
\hline 9. ca_ancora & 85.47 & UDPipe Future & 72.60 & \pm 12.31 \\
\hline 10. es_ancora & 84.92 & HIT-SCIR & 72.10 & \pm 12.71 \\
\hline 11. it_isdt & 84.76 & ICS PAS & 75.42 & \pm 10.72 \\
\hline 12. fr_sequoia & 84.67 & ICS PAS & 70.63 & \pm 11.66 \\
\hline 13. no_nynorsk & 84.44 & TurkuNLP & 67.43 & \pm 14.10 \\
\hline 14. la_ittb & 84.37 & TurkuNLP & 68.10 & \pm 17.85 \\
\hline 15. bg_btb & 84.31 & TurkuNLP & 68.13 & \pm 15.02 \\
\hline 16. fro_srcmf & 84.11 & UDPipe Future & 70.46 & \pm 16.40 \\
\hline 17. sr_set & 83.28 & TurkuNLP & 65.62 & \pm 17.61 \\
\hline 18. sl_ssj & 83.23 & Stanford & 62.54 & \pm 17.20 \\
\hline 19. fi_ftb & 82.44 & TurkuNLP & 59.66 & \pm 16.50 \\
\hline 20. fi_pud & 82.44 & TurkuNLP & 52.25 & \pm 18.50 \\
\hline 21. sv_talbanken & 81.44 & TurkuNLP & 66.45 & \pm 13.18 \\
\hline 22. fi_tdt & 81.24 & TurkuNLP & 54.70 & \pm 17.25 \\
\hline 23. fr_gsd & 81.18 & HIT-SCIR & 69.61 & \pm 10.58 \\
\hline 24. ro_rrt & 80.97 & TurkuNLP & 63.53 & \pm 15.84 \\
\hline 25. sk_snk & 80.74 & TurkuNLP & 58.35 & \pm 15.07 \\
\hline 26. pt_bosque & 80.62 & TurkuNLP & 68.71 & \pm 11.27 \\
\hline 27. en_pud & 80.53 & LATTICE & 64.73 & \pm 10.88 \\
\hline 28. cs_pud & 80.53 & ICS PAS & 64.62 & \pm 16.03 \\
\hline 29. hr_set & 80.50 & TurkuNLP & 64.64 & \pm 17.13 \\
\hline 30. fa_seraji & 80.44 & Stanford & 68.38 & \pm 7.39 \\
\hline 31. el_gdt & 80.09 & TurkuNLP & 63.26 & \pm 15.60 \\
\hline 32. ko_kaist & 79.55 & TurkuNLP & 57.32 & \pm 20.78 \\
\hline 33. et_edt & 79.37 & TurkuNLP & 57.06 & \pm 16.14 \\
\hline 34. nl_alpino & 79.15 & HIT-SCIR & 64.29 & \pm 10.83 \\
\hline 35. en_ewt & 78.44 & HIT-SCIR & 67.53 & \pm 8.47 \\
\hline 36. uk_iu & 78.38 & TurkuNLP & 57.78 & \pm 15.95 \\
\hline 37. eu_bdt & 78.15 & TurkuNLP & 60.52 & \pm 15.24 \\
\hline 38. da_ddt & 78.07 & TurkuNLP & 63.16 & \pm 11.41 \\
\hline 39. sv_lines & 77.01 & ICS PAS & 63.13 & \pm 11.72 \\
\hline 40. id_gsd & 76.56 & Stanford & 62.52 & \pm 7.89 \\
\hline 41. nl_lassysmall & 76.54 & HIT-SCIR & 60.92 & \pm 11.93 \\
\hline 42. af_afribooms & 76.44 & TurkuNLP & 63.87 & \pm 9.62 \\
\hline 43. ko_gsd & 76.31 & TurkuNLP & 54.13 & \pm 17.78 \\
\hline 44. en_lines & 75.29 & HIT-SCIR & 62.29 & \pm 9.27 \\
\hline 45. gl_ctg & 75.14 & Stanford & 60.86 & \pm 10.82 \\
\hline 46. ur_udtb & 73.79 & TurkuNLP & 62.93 & \pm 6.42 \\
\hline 47. ja_gsd & 73.79 & HIT-SCIR & 60.87 & \pm 6.04 \\
\hline 48. en_gum & 73.57 & ICS PAS & 61.02 & \pm 8.59 \\
\hline 49. hu_szeged & 73.17 & TurkuNLP & 55.42 & \pm 10.95 \\
\hline 50. $\mathrm{zh}_{-} \mathrm{gsd}$ & 72.97 & HIT-SCIR & 55.66 & \pm 6.26 \\
\hline 51. lv_lvtb & 72.40 & TurkuNLP & 53.42 & \pm 14.56 \\
\hline 52. de_gsd & 71.40 & HIT-SCIR & 54.86 & \pm 14.99 \\
\hline 53. cu_proiel & 71.31 & Stanford & |51.27 & \pm 15.35 \\
\hline 54. ar_padt & 70.06 & Stanford & 49.13 & \pm 18.98 \\
\hline 55. it_postwita & 69.34 & HIT-SCIR & 50.97 & \pm 8.76 \\
\hline 56. grc_proiel & 69.03 & TurkuNLP & 48.58 & \pm 19.91 \\
\hline 57. la_proiel & 67.60 & TurkuNLP & 51.03 & \pm 14.56 \\
\hline 58. sv_pud & 66.12 & TurkuNLP & 50.20 & \pm 11.30 \\
\hline 59. fr_spoken & 65.63 & HIT-SCIR & 52.57 & \pm 7.29 \\
\hline 60. he_htb & 65.04 & Stanford & 47.22 & \pm 6.60 \\
\hline 61. ru_taiga & 64.36 & ICS PAS & 39.32 & \pm 10.49 \\
\hline 62. gl_treegal & 64.29 & UDPipe Future & 49.38 & \pm 8.18 \\
\hline 63. got_proiel & 63.98 & Stanford & 48.79 & \pm 13.77 \\
\hline 64. no_nynorsklia & 60.98 & ICS PAS & 41.20 & \pm 8.64 \\
\hline 65. tr_imst & 60.13 & TurkuNLP & 45.39 & \pm 10.38 \\
\hline 66. sme_giella & 60.10 & TurkuNLP & 35.76 & \pm 12.68 \\
\hline 67. grc_perseus & 58.68 & TurkuNLP & 36.48 & \pm 16.03 \\
\hline 68. ug_udt & 55.42 & HIT-SCIR & 41.64 & \pm 8.09 \\
\hline 69. ga_idt & 55.18 & TurkuNLP & 37.83 & \pm 7.61 \\
\hline 70. la_perseus & 52.75 & ICS PAS & 30.16 & \pm 11.05 \\
\hline 71. sl_sst & 50.94 & ICS PAS & 37.20 & \pm 6.87 \\
\hline 72. vi_vtb & 44.02 & Stanford & 35.50 & \pm 3.74 \\
\hline 73. pem_nsc & 26.04 & CUNI $x$-ling & 12.07 & \pm 5.63 \\
\hline 74. hsb_ufal & 21.09 & LATTICE & 11.26 & \pm 4.97 \\
\hline 75. br_keb & 20.70 & TurkuNLP & 4.19 & \pm 4.93 \\
\hline 76. hy_armtdp & 19.04 & CUNI $x$-ling & 10.68 & \pm 4.37 \\
\hline 77. fo_oft & 14.40 & CUNI $x$-ling & 7.32 & \pm 3.33 \\
\hline 78. ja_modern & 13.79 & Stanford & 7.70 & \pm 2.86 \\
\hline 79. kmr_mg & 13.66 & LATTICE & 8.44 & \pm 3.11 \\
\hline 80. kk_ktb & 11.33 & CUNI $x$-ling & 6.75 & \pm 2.95 \\
\hline 81. th_pud & 10.77 & CUNI $x$-ling & 0.91 & \pm 2.11 \\
\hline 82. bxr_bdt & 6.65 & AntNLP & 3.39 & \pm 1.61 \\
\hline
\end{tabular}

Table 16: Treebank ranking by best parser MLAS. Table 17: Treebank ranking by best parser BLEX. 


\begin{tabular}{|c|c|c|c|c|}
\hline Treebank & LAS & MLAS & Diff & Language \\
\hline 1. de_gsd & 70.13 & 39.13 & 31.01 & German \\
\hline 2. sv_pud & 67.02 & 39.41 & 27.61 & Swedish \\
\hline 3. fo_oft & 27.87 & 0.37 & 27.50 & Faroese \\
\hline 4. ur_udtb & 75.89 & 49.64 & 26.25 & Urdu \\
\hline 5. ga_idt & 58.37 & 33.70 & 24.66 & Irish \\
\hline 6. grc_perseus & 59.01 & 35.65 & 23.36 & Ancient Greek \\
\hline 7. hsb_ufal & 26.48 & 4.66 & 21.82 & Upper Sorbian \\
\hline 8. sk_snk & 76.53 & 56.82 & 19.71 & Slovak \\
\hline 9. ug_udt & 54.27 & 35.08 & 19.20 & Uyghur \\
\hline 10. ru_taiga & 56.27 & 37.16 & 19.12 & Russian \\
\hline 11. hr_set & 78.37 & 60.08 & 18.29 & Croatian \\
\hline 12. la_perseus & 46.91 & 28.67 & 18.24 & Latin \\
\hline 13. grc_proiel & 65.02 & 47.62 & 17.40 & Ancient Greek \\
\hline 14. gl_treegal & 64.65 & 47.35 & 17.30 & Galician \\
\hline 15. uk_iu & 72.47 & 55.45 & 17.01 & Ukrainian \\
\hline 16. hi_hdtb & 85.16 & 68.48 & 16.68 & Hindi \\
\hline 17. pl_sz & 81.47 & 64.80 & 16.67 & Polish \\
\hline 18. hy_armtdp & 22.39 & 5.94 & 16.45 & Armenian \\
\hline 19. el_gdt & 80.65 & 64.29 & 16.36 & Greek \\
\hline 20. sv_lines & 73.76 & 57.40 & 16.36 & Swedish \\
\hline 21. $\mathrm{kmr} \_\mathrm{mg}$ & 20.27 & 4.01 & 16.26 & Kurmanji \\
\hline 22. nl_alpino & 77.76 & 62.82 & 14.95 & Dutch \\
\hline 23. gl_ctg & 72.46 & 57.92 & 14.55 & Galician \\
\hline 24. $1 \mathrm{v} \_l v t b$ & 67.76 & 53.31 & 14.45 & Latvian \\
\hline 25. got_proiel & 60.55 & 46.18 & 14.37 & Gothic \\
\hline 26. pt_bosque & 80.49 & 66.22 & 14.27 & Portuguese \\
\hline 27. ja_gsd & 73.68 & 59.52 & 14.16 & Japanese \\
\hline 28. kk_ktb & 19.11 & 5.04 & 14.07 & Kazakh \\
\hline 29. hu_szeged & 67.05 & 53.08 & 13.96 & Hungarian \\
\hline 30. sl_sst & 47.07 & 33.12 & 13.95 & Slovenian \\
\hline 31. eu_bdt & 72.08 & 58.49 & 13.59 & Basque \\
\hline 32. he_htb & 58.73 & 45.22 & 13.51 & Hebrew \\
\hline 33. la_proiel & 61.25 & 47.79 & 13.46 & Latin \\
\hline 34. no_nynorsklia & 50.33 & 37.08 & 13.25 & Norwegian \\
\hline 35. it_postwita & 64.95 & 51.72 & 13.22 & Italian \\
\hline 36. nl_lassysmall & 75.08 & 61.95 & 13.14 & Dutch \\
\hline 37. af_afribooms & 76.61 & 63.76 & 12.84 & Afrikaans \\
\hline 38. sme_giella & 51.10 & 38.29 & 12.82 & North Sámi \\
\hline 39. cs_pud & 73.24 & 60.47 & 12.77 & Czech \\
\hline 40. sl_ssj & 75.00 & 62.41 & 12.59 & Slovenian \\
\hline 41. sr_set & 79.84 & 67.33 & 12.50 & Serbian \\
\hline 42. en_gum & 74.20 & 61.72 & 12.48 & English \\
\hline 43. ja_modern & 18.92 & 6.45 & 12.47 & Japanese \\
\hline 44. cu_proiel & 62.64 & 50.28 & 12.36 & Old Church Slavonic \\
\hline 45. cs_fictree & 82.10 & 69.91 & 12.19 & Czech \\
\hline 46. pl_lfg & 85.89 & 73.73 & 12.17 & Polish \\
\hline 47. id_gsd & 73.05 & 61.03 & 12.02 & Indonesian \\
\hline 48. br_keb & 13.27 & 1.52 & 11.75 & Breton \\
\hline 49. fr_spoken & 64.66 & 53.17 & 11.49 & French \\
\hline 50. en_pud & 74.51 & 63.05 & 11.46 & English \\
\hline 51. cs_cac & 82.69 & 71.39 & 11.29 & Czech \\
\hline 52. ar_padt & 64.07 & 53.28 & 10.79 & Arabic \\
\hline 53. fi_ftb & 76.89 & 66.11 & 10.78 & Finnish \\
\hline 54. it_isdt & 87.61 & 77.14 & 10.47 & Italian \\
\hline 55. tr_imst & 55.61 & 45.26 & 10.34 & Turkish \\
\hline 56. $\mathrm{pcm} \_n s c$ & 13.19 & 3.00 & 10.19 & Naija \\
\hline 57. fr_sequoia & 80.55 & 70.42 & 10.13 & French \\
\hline 58. bxr_bdt & 11.45 & 1.33 & 10.12 & Buryat \\
\hline 59. fr_gsd & 79.43 & 69.33 & 10.10 & French \\
\hline 60. da_ddt & 75.02 & 65.00 & 10.02 & Danish \\
\hline 61. no_nynorsk & 78.55 & 68.62 & 9.93 & Norwegian \\
\hline 62. en_lines & 72.28 & 62.35 & 9.93 & English \\
\hline 63. $\mathrm{zh}_{\text {_gsd }}$ & 60.32 & 50.42 & 9.90 & Chinese \\
\hline 64. sv_talbanken & 77.71 & 68.05 & 9.66 & Swedish \\
\hline 65. bg_btb & 82.52 & 73.18 & 9.34 & Bulgarian \\
\hline 66. la_ittb & 77.00 & 67.77 & 9.23 & Latin \\
\hline 67. fro_sremf & 74.38 & 65.19 & 9.18 & Old French \\
\hline 68. en_ewt & 75.99 & 66.84 & 9.15 & English \\
\hline 69. no_bokmaal & 79.80 & 70.75 & 9.05 & Norwegian \\
\hline 70. ca_ancora & 83.61 & 74.62 & 8.99 & Catalan \\
\hline 71. cs_pdt & 82.18 & 73.61 & 8.57 & Czech \\
\hline 72. et_edt & 72.08 & 63.59 & 8.50 & Estonian \\
\hline 73. ro_rrt & 75.77 & 67.43 & 8.33 & Romanian \\
\hline 74. fi_tdt & 73.55 & 65.27 & 8.28 & Finnish \\
\hline 75. es_ancora & 82.84 & 74.61 & 8.23 & Spanish \\
\hline 76. ko_gsd & 71.88 & 63.73 & 8.15 & Korean \\
\hline 77. ru_syntagrus & 79.68 & 71.63 & 8.05 & Russian \\
\hline 78. vi_vtb & 40.40 & 32.45 & 7.95 & Vietnamese \\
\hline 79. fa_seraji & 78.71 & 71.23 & 7.48 & Persian \\
\hline 80. ko_kaist & 77.10 & 70.18 & 6.92 & Korean \\
\hline 81. fi_pud & 68.87 & 62.38 & 6.49 & Finnish \\
\hline 82. th_pud & 1.38 & 0.42 & 0.96 & Thai \\
\hline
\end{tabular}

Table 18: Treebank ranking by difference between average parser LAS and MLAS.

\begin{tabular}{|l|r|l|r|r|}
\hline \multicolumn{1}{|c|}{ Treebank } & Best & Best system & Avg & StDev \\
\hline 70. bxr_bdt & 99.24 & IBM NY & 88.64 & \pm 8.09 \\
71. fi_pud & 99.69 & Uppsala & 88.13 & \pm 10.81 \\
72. zh_gsd & 96.71 & HIT-SCIR & 86.91 & \pm 3.83 \\
73. fo_oft & 99.47 & CUNI x-ling & 86.76 & \pm 10.68 \\
74. ar_padt & 96.81 & Stanford & 86.62 & \pm 7.00 \\
75. kmr_mg & 96.97 & Uppsala & 86.61 & \pm 7.16 \\
76. kk_ktb & 97.40 & Uppsala & 85.55 & \pm 7.45 \\
77. br_keb & 92.45 & TurkuNLP & 83.76 & \pm 7.37 \\
78. he_htb & 93.98 & Stanford & 82.45 & \pm 3.80 \\
79. vi_vtb & 93.46 & HIT-SCIR & 81.71 & \pm 3.73 \\
80. pcm_nsc & 99.71 & CEA LIST & 79.94 & \pm 10.69 \\
81. ja_modern & 75.69 & HIT-SCIR & 59.40 & \pm 7.70 \\
82. th_pud & 69.93 & Uppsala & 17.16 & \pm 20.57 \\
\hline
\end{tabular}

Table 19: Treebanks with most difficult word segmentation (by average parser $\mathrm{F}_{1}$ ).

\begin{tabular}{|l|r|l|r|r|}
\hline \multicolumn{1}{|c|}{ Treebank } & Best & Best system & Avg & StDev \\
\hline 73. grc_proiel & 51.84 & HIT-SCIR & 42.46 & \pm 7.33 \\
74. cu_proiel & 48.67 & Stanford & 35.54 & \pm 4.02 \\
75. la_proiel & 39.61 & Stanford & 33.40 & \pm 5.39 \\
76. got_proiel & 38.23 & Stanford & 27.22 & \pm 4.47 \\
77. it_postwita & 65.90 & Stanford & 25.25 & \pm 14.30 \\
78. sl_sst & 24.43 & NLP-Cube & 20.92 & \pm 4.70 \\
79. fr_spoken & 24.17 & Stanford & 20.43 & \pm 2.89 \\
80. th_pud & 12.37 & TurkuNLP & 1.75 & \pm 3.68 \\
81. pcm_nsc & 0.93 & Stanford & 0.06 & \pm 0.19 \\
82. ja_modern & 0.23 & Stanford & 0.01 & \pm 0.04 \\
\hline
\end{tabular}

Table 20: Treebanks with most difficult sentence segmentation (by average parser $\mathrm{F}_{1}$ ).

\section{Analysis of Submitted Systems}

Table 21 gives an overview of 24 of the systems evaluated in the shared task. The overview is based on a post-evaluation questionnaire to which 24 of 25 teams responded. Systems are ordered alphabetically by name and their LAS rank is indicated in the second column.

Looking first at word and sentence segmentation, we see that, while a clear majority of systems $(19 / 24)$ rely on the baseline system for segmentation, slightly more than half $(13 / 24)$ have developed their own segmenter, or tuned the baseline segmenter, for at least a subset of languages. This is a development from 2017, where only 7 out of 29 systems used anything other than the baseline segmenter.

When it comes to morphological analysis, including universal POS tags, features and lemmas, all systems this year include some such component, and only 6 systems rely entirely on the base- 


\begin{tabular}{|c|c|c|c|c|c|c|c|}
\hline System & $\mathbf{R}$ & Segment & Morph & Syntax & WEmb & Additional Data & MultiLing \\
\hline AntNLP & 9 & Base & Base & Single-G & FB & None & $\mathrm{Own}_{\mathrm{S}}$ \\
\hline ArmParser & 25 & Base & Own & Single & FB & None & None \\
\hline BOUN & 21 & Base & Base & Single-T & Base & None & None \\
\hline CEA LIST & 6 & Base & $\mathrm{B}_{\mathrm{L}} / \mathrm{Own}$ & Single-G/T & $\mathrm{B} / \mathrm{FB}$ & OPUS/Wikt & $\mathrm{Own}_{\mathrm{L}}$ \\
\hline CUNI x-ling & 20 & $\mathrm{~B} / \mathrm{Own}$ & B/Own & Single/Ens & FB/None & O/UM/WALS/W & $\mathrm{Own}_{\mathrm{L}, \mathrm{S}}$ \\
\hline Fudan & 17 & Base & Base & Ensemble & None & None & $\mathrm{Own}_{\mathrm{L}, \mathrm{S}}$ \\
\hline HIT-SCIR & 1 & B/Own & Base & Ensemble & B/FB/Crawl & None & $\mathrm{Own}_{\mathrm{L}, \mathrm{S}}$ \\
\hline HUJI & 24 & Base & Base & Single-T & FB & None & $\mathrm{Own}_{\mathrm{L}}$ \\
\hline IBM NY & 13 & $\mathrm{~B} / \mathrm{Own}$ & B/Joint & Ensemble-T & $\mathrm{B} / \mathrm{FB}$ & Wiki & $\mathrm{Own}_{\mathrm{L}, \mathrm{S}}$ \\
\hline ICS PAS & 3 & Base & Own & Single-G & FB/None & None & None \\
\hline KParse & 16 & $\mathrm{~B} / \mathrm{Own}$ & Own & Single & Other & None & $\mathrm{Own}_{\mathrm{L}}$ \\
\hline LATTICE & 3 & Base & $\mathrm{Own}_{\mathrm{U}}$ & Single-G/Ens & B/FB/Crawl & OPUS/Wiki & $\mathrm{Own}_{\mathrm{L}, \mathrm{S}}$ \\
\hline LeisureX & 15 & Base & Own & Single & Base & None & $\mathrm{Own}_{\mathrm{L}}$ \\
\hline NLP-Cube & 9 & Own & Own & Single & FB & None & $\mathrm{Own}_{\mathrm{L}}$ \\
\hline ONLP lab & 22 & Base & Base & Single-T & None & UML & None \\
\hline ParisNLP & 11 & $\mathrm{~B} / \mathrm{Own}$ & B/Own & Single-G & FB & UML & $\mathrm{Own}_{\mathrm{L}}$ \\
\hline Phoenix & 19 & Own & $\mathrm{Own}_{\mathrm{U}}$ & Single & Train & None & $\mathrm{Own}_{\mathrm{L}}$ \\
\hline SLT-Interactions & 12 & B/Own & Own & Single & Crawl & None & $\mathrm{Own}_{\mathrm{L}}$ \\
\hline SParse & 26 & $\mathrm{~B} / \mathrm{Own}$ & Own & Single-G & Crawl & None & $\mathrm{Own}_{\mathrm{L}}$ \\
\hline Stanford & 7 & Own & Own & Single-G & $\mathrm{B} / \mathrm{FB}$ & None & None \\
\hline TurkuNLP & 2 & $\mathrm{~B} / \mathrm{Own}$ & Own & Single-G & $\mathrm{B} / \mathrm{FB}$ & OPUS/Aper & $\mathrm{Own}_{\mathrm{L}}$ \\
\hline UDPipe Future & 3 & Own & Joint & Single-G & $\mathrm{B} / \mathrm{FB}$ & None & None \\
\hline UniMelb & 14 & Base & Joint & Single & Base & None & Base \\
\hline Uppsala & 7 & Own & $\mathrm{Own}_{\mathrm{U}, \mathrm{F}}$ & Single-T & B/FB/Wiki & OPUS/Wiki/Aper & $\mathrm{Own}_{\mathrm{L}, \mathrm{S}}$ \\
\hline
\end{tabular}

Table 21: Classification of participating systems. $\mathbf{R}=$ LAS ranking. Segment $=$ word/sentence segmentation. Morph $=$ morphological analysis, including universal POS tags $[\mathrm{U}]$, features $[\mathrm{F}]$ and lemmas $[\mathrm{L}]$, with subscripts for subsets [Joint $=$ morphological component trained jointly with syntactic parser]. Syntax $=$ syntactic parsing [Single $=$ single parser; Ensemble $($ or Ens $)=$ parser ensemble; $\mathrm{G}=$ graph-based; $\mathrm{T}=$ transition-based]. WEmb $=$ pre-trained word embeddings [FB $=$ Facebook; Crawl $=$ trained on web crawl data provided by the organizers; Wiki $=$ trained on Wikipedia data; Train $=$ trained on treebank training data]. Additional Data $=$ data used in addition to treebank training sets [OPUS (or O) $=$ OPUS, Aper $=$ Apertium morphological analysers, Wikt $=$ Wiktionary, Wiki $=$ Wikipedia, $\mathrm{UM}=$ UniMorph, $\mathrm{UML}=$ Universal Morphological Lattices, WALS $=$ World Atlas of Language Structures]. MultiLing = multilingal models used for low-resource (L) or small (S) languages. In all columns, Base (or B) refers to the Baseline UDPipe system or the baseline word embeddings provided by the organizers, while None means that there is no corresponding component in the system.

line UDPipe system. This is again quite different from 2017, where more than half the systems either just relied on the baseline tagger (13 systems) or did not predict any morphology at all (3 systems). We take this to be primarily a reflection of the fact that two out of three official metrics included (some) morphological analysis this year, although 3 systems did not predict the lemmas required for the BLEX metric (and 2 systems only predicted universal POS tags, no features). As far as we can tell from the questionnaire responses, only 3 systems used a model where morphology and syntax were predicted jointly. ${ }^{14}$

For syntactic parsing, most teams (19) use a single parsing model, while 5 teams, including the winning HIT-SCIR system, build ensemble models, either for all languages or a subset of them. When it comes to the type of parsing model, we observe that graph-based models are more popular than transition-based models this year, while the opposite was true in 2017 . We hypothesize that

\footnotetext{
${ }^{14}$ The ONLP lab system also has a joint model but in the end used the baseline morphology as it gave better results.
} 
this is due to the superior performance of the Stanford graph-based parser in last year's shared task, and many of the high-performing systems this year either incorporate that parser or a reimplementation of it. ${ }^{15}$

The majority of parsers make use of pre-trained word embeddings. Most popular are the Facebook embeddings, which are used by 17 systems, followed by the baseline embeddings provided by the organizers (11), and embeddings trained on web crawl data (4). ${ }^{16}$ When it comes to additional data, over and above the treebank training sets and pretrained word embeddings, the most striking observation is that a majority of systems (16) did not use any at all. Those that did primarily used OPUS (5), Wikipedia dumps (3), Apertium morphological analyzers (2), and Universal Morphological Lattices (2). The CUNI x-ling system, which focused on low-resource languages, also exploited UniMorph and WALS (in addition to OPUS and Wikipedia).

Finally, we note that a majority of systems make use of models trained on multiple languages to improve parsing for languages with little or no training data. According to the questionnaire responses, 15 systems use multilingual models for the languages classified as "low-resource", while 7 systems use them for the languages classified as "small". ${ }^{17}$ Only one system relied on the baseline delexicalized parser trained on data from all languages.

\section{Conclusion}

The CoNLL 2018 Shared Task on UD parsing, the second in the series, was novel in several respects. Besides using cross-linguistically consistent linguistic representations, emphasizing end-to-end processing of text, and in using a multiply parallel test set, as in 2017, it was unusual also in featuring an unprecedented number of languages and treebanks and in integrating cross-lingual learning for resource-poor languages. Compared to the first edition of the task in 2017, this year several languages were provided with little-to-no resources, whereas in 2017, predicted morphology trained on

\footnotetext{
${ }^{15}$ This is true of at least 3 of the 5 best performing systems.

${ }^{16}$ The baseline embeddings were the same as in 2017 and therefore did not cover new languages, which may partly explain the greater popularity of the Facebook embeddings this year.

${ }^{17}$ We know that some teams used them also for clusters involving high-resource languages, but we have no detailed statistics on this usage.
}

the language in question was available for all of the languages. The most extreme example of these is Thai, where the only accessible resource was the Facebook Research Thai embeddings model and the OPUS parallel corpora. This year's task also introduced two additional metrics that take into account morphology and lemmatization. This encouraged the development of truly end-to-end full parsers, producing complete parses including morphological features and lemmas in addition to the syntactic tree. This also aimed to improve the utility of the systems developed in the shared task for later downstream applications. For most UD languages, these parsers represent a new state of the art for end-to-end dependency parsing.

The analysis of the shared task results has so far only scratched the surface, and we refer to the system description papers for more in-depth analysis of individual systems and their performance. For many previous CoNLL shared tasks, the task itself has only been the starting point of a long and fruitful research strand, enabled by the resources created for the task. We hope and believe that the 2017 and 2018 UD parsing tasks will join this tradition.

\section{Acknowledgments}

We are grateful to all the contributors to Universal Dependencies; without their effort a task like this simply wouldn't be possible.

The work described herein, including data preparation for the CoNLL 2018 UD Shared Task, has been supported by the following grants and projects: OP PPR No. CZ.07.1.02/0.0/0.0/16_023/0000108 of the City of Prague, "CRACKER," H2020 Project No. 645357 of the European Commission; "MANYLA," Grant No. GA15-10472S of the Grant Agency of the Czech Republic; FIN-CLARIN; Grant No. 2016-01817 of the Swedish Research Council; and the LINDAT/CLARIN research infrastructure project funded by the Ministry of Education, Youth and Sports of the Czech Republic, Project. No. LM2015071. The data for the CoNLL 2018 UD Shared Task are available also via the LINDAT/CLARIN repository.

\section{References}

Gor Arakelyan, Karen Hambardzumyan, and Hrant Khachatrian. 2018. Towards JointUD: Part-ofspeech tagging and lemmatization using recurrent 
neural networks. In Proceedings of the CoNLL 2018 Shared Task: Multilingual Parsing from Raw Text to Universal Dependencies. Association for Computational Linguistics.

Riyaz Ahmad Bhat, Irshad Ahmad Bhat, and Srinivas Bangalore. 2018. The SLT-Interactions parsing system at the CoNLL 2018 shared task. In Proceedings of the CoNLL 2018 Shared Task: Multilingual Parsing from Raw Text to Universal Dependencies. Association for Computational Linguistics.

Piotr Bojanowski, Edouard Grave, Armand Joulin, and Tomas Mikolov. 2016. Enriching word vectors with subword information arXiv preprint arXiv:1607.04606.

Tiberiu Boros, Stefan Daniel Dumitrescu, and Ruxandra Burtica. 2018. NLP-Cube: End-to-end raw text processing with neural networks. In Proceedings of the CoNLL 2018 Shared Task: Multilingual Parsing from Raw Text to Universal Dependencies. Association for Computational Linguistics.

Sabine Buchholz and Erwin Marsi. 2006. CoNLL-X shared task on multilingual dependency parsing. In Proceedings of the 10th Conference on Computational Natural Language Learning (CoNLL-X). Association for Computational Linguistics, pages 149164. http://anthology.aclweb.org/W/W06/W0629.pdf\#page $=165$.

Wanxiang Che, Yijia Liu, Yuxuan Wang, Bo Zheng, and Ting Liu. 2018. Towards better UD parsing: Deep contextualized word embeddings, ensemble, and treebank concatenation. In Proceedings of the CoNLL 2018 Shared Task: Multilingual Parsing from Raw Text to Universal Dependencies. Association for Computational Linguistics.

Danlu Chen, Mengxiao Lin, Zhifeng Hu, and Xipeng Qiu. 2018. A simple yet effective joint training method for cross-lingual universal dependency parsing. In Proceedings of the CoNLL 2018 Shared Task: Multilingual Parsing from Raw Text to Universal Dependencies. Association for Computational Linguistics.

Elie Duthoo and Olivier Mesnard. 2018. CEA LIST : Processing low-resource languages for CoNLL 2018. In Proceedings of the CoNLL 2018 Shared Task: Multilingual Parsing from Raw Text to Universal Dependencies. Association for Computational Linguistics.

Murhaf Fares, Stephan Oepen, Lilja Øvrelid, Jari Björne, and Richard Johansson. 2018. The 2018 shared task on extrinsic parser evaluation: On the downstream utility of English universal dependency parsers. In Proceedings of the CoNLL 2018 Shared Task: Multilingual Parsing from Raw Text to Universal Dependencies. Association for Computational Linguistics, Brussels, Belgium.
Filip Ginter, Jan Hajič, Juhani Luotolahti, Milan Straka, and Daniel Zeman. 2017. CoNLL 2017 shared task automatically annotated raw texts and word embeddings. LINDAT/CLARIN digital library at the Institute of Formal and Applied Linguistics, Charles University. http://hdl.handle.net/11234/1-1989.

Allan Hanbury, Henning Müller, Krisztian Balog, Torben Brodt, Gordon V. Cormack, Ivan Eggel, Tim Gollub, Frank Hopfgartner, Jayashree KalpathyCramer, Noriko Kando, Anastasia Krithara, Jimmy Lin, Simon Mercer, and Martin Potthast. 2015. Evaluation-as-a-Service: Overview and Outlook. ArXiv e-prints http://arxiv.org/abs/1512.07454.

Daniel Hershcovich, Omri Abend, and Ari Rappoport. 2018. Universal dependency parsing with a general transition-based DAG parser. In Proceedings of the CoNLL 2018 Shared Task: Multilingual Parsing from Raw Text to Universal Dependencies. Association for Computational Linguistics.

Ganesh Jawahar, Benjamin Muller, Amal Fethi, Louis Martin, Éric de La Clergerie, Benoît Sagot, and Djamé Seddah. 2018. ELMoLex: Connecting ELMo and lexicon features for dependency parsing. In Proceedings of the CoNLL 2018 Shared Task: Multilingual Parsing from Raw Text to Universal Dependencies. Association for Computational Linguistics.

Tao Ji, Yufang Liu, Yijun Wang, Yuanbin Wu, and Man Lan. 2018. AntNLP at CoNLL 2018 shared task: A graph-based parser for universal dependency parsing. In Proceedings of the CoNLL 2018 Shared Task: Multilingual Parsing from Raw Text to Universal Dependencies. Association for Computational Linguistics.

Jenna Kanerva, Filip Ginter, Niko Miekka, Akseli Leino, and Tapio Salakoski. 2018. Turku neural parser pipeline: An end-to-end system for the CoNLL 2018 shared task. In Proceedings of the CoNLL 2018 Shared Task: Multilingual Parsing from Raw Text to Universal Dependencies. Association for Computational Linguistics.

Ömer Kırnap, Erenay Dayanık, and Deniz Yuret. 2018. Tree-stack LSTM in transition based dependency parsing. In Proceedings of the CoNLL 2018 Shared Task: Multilingual Parsing from Raw Text to Universal Dependencies. Association for Computational Linguistics.

Zuchao Li, Shexia He, Zhuosheng Zhang, and Hai Zhao. 2018. Joint learning of pos and dependencies for multilingual universal dependency parsing. In Proceedings of the CoNLL 2018 Shared Task: Multilingual Parsing from Raw Text to Universal Dependencies. Association for Computational Linguistics.

KyungTae Lim, Cheoneum Park, Changki Lee, and Thierry Poibeau. 2018. SEx BiST: A multi-source trainable parser with deep contextualized lexical representations. In Proceedings of the CoNLL 2018 
Shared Task: Multilingual Parsing from Raw Text to Universal Dependencies. Association for Computational Linguistics.

Dat Quoc Nguyen and Karin Verspoor. 2018. An improved neural network model for joint pos tagging and dependency parsing. In Proceedings of the CoNLL 2018 Shared Task: Multilingual Parsing from Raw Text to Universal Dependencies. Association for Computational Linguistics.

Joakim Nivre, Mitchell Abrams, Željko Agić, Lars Ahrenberg, Lene Antonsen, Maria Jesus Aranzabe, Gashaw Arutie, Masayuki Asahara, Luma Ateyah, Mohammed Attia, Aitziber Atutxa, Liesbeth Augustinus, Elena Badmaeva, Miguel Ballesteros, Esha Banerjee, Sebastian Bank, Verginica Barbu Mititelu, John Bauer, Sandra Bellato, Kepa Bengoetxea, Riyaz Ahmad Bhat, Erica Biagetti, Eckhard Bick, Rogier Blokland, Victoria Bobicev, Carl Börstell, Cristina Bosco, Gosse Bouma, Sam Bowman, Adriane Boyd, Aljoscha Burchardt, Marie Candito, Bernard Caron, Gauthier Caron, Gülşen Cebiroğlu Eryiğit, Giuseppe G. A. Celano, Savas Cetin, Fabricio Chalub, Jinho Choi, Yongseok Cho, Jayeol Chun, Silvie Cinková, Aurélie Collomb, Çağrı Çöltekin, Miriam Connor, Marine Courtin, Elizabeth Davidson, Marie-Catherine de Marneffe, Valeria de Paiva, Arantza Diaz de Ilarraza, Carly Dickerson, Peter Dirix, Kaja Dobrovoljc, Timothy Dozat, Kira Droganova, Puneet Dwivedi, Marhaba Eli, Ali Elkahky, Binyam Ephrem, Tomaž Erjavec, Aline Etienne, Richárd Farkas, Hector Fernandez Alcalde, Jennifer Foster, Cláudia Freitas, Katarína Gajdošová, Daniel Galbraith, Marcos Garcia, Moa Gärdenfors, Kim Gerdes, Filip Ginter, Iakes Goenaga, Koldo Gojenola, Memduh Gökırmak, Yoav Goldberg, Xavier Gómez Guinovart, Berta Gonzáles Saavedra, Matias Grioni, Normunds Grūzītis, Bruno Guillaume, Céline Guillot-Barbance, Nizar Habash, Jan Hajič, Jan Hajič jr., Linh Hà Mỹ, Na-Rae Han, Kim Harris, Dag Haug, Barbora Hladká, Jaroslava Hlaváčová, Florinel Hociung, Petter Hohle, Jena Hwang, Radu Ion, Elena Irimia, Tomáš Jelínek, Anders Johannsen, Fredrik Jørgensen, Hüner Kaşıkara, Sylvain Kahane, Hiroshi Kanayama, Jenna Kanerva, Tolga Kayadelen, Václava Kettnerová, Jesse Kirchner, Natalia Kotsyba, Simon Krek, Sookyoung Kwak, Veronika Laippala, Lorenzo Lambertino, Tatiana Lando, Septina Dian Larasati, Alexei Lavrentiev, John Lee, Phương Lê Hồng, Alessandro Lenci, Saran Lertpradit, Herman Leung, Cheuk Ying Li, Josie Li, Keying Li, KyungTae Lim, Nikola Ljubešić, Olga Loginova, Olga Lyashevskaya, Teresa Lynn, Vivien Macketanz, Aibek Makazhanov, Michael Mandl, Christopher Manning, Ruli Manurung, Cătălina Mărănduc, David Mareček, Katrin Marheinecke, Héctor Martínez Alonso, André Martins, Jan Mašek, Yuji Matsumoto, Ryan McDonald, Gustavo Mendonça, Niko Miekka, Anna Missilä, Cătălin Mititelu, Yusuke Miyao, Simonetta Montemagni, Amir More, Laura Moreno Romero, Shinsuke Mori, Bjartur Mortensen, Bohdan Moskalevskyi,
Kadri Muischnek, Yugo Murawaki, Kaili Müürisep, Pinkey Nainwani, Juan Ignacio Navarro Horñiacek, Anna Nedoluzhko, Gunta Nešpore-Bērzkalne, Lương Nguyễn Thị, Huyền Nguyễn Thị Minh, Vitaly Nikolaev, Rattima Nitisaroj, Hanna Nurmi, Stina Ojala, Adédayọ̀ Olúòkun, Mai Omura, Petya Osenova, Robert Östling, Lilja Øvrelid, Niko Partanen, Elena Pascual, Marco Passarotti, Agnieszka Patejuk, Siyao Peng, Cenel-Augusto Perez, Guy Perrier, Slav Petrov, Jussi Piitulainen, Emily Pitler, Barbara Plank, Thierry Poibeau, Martin Popel, Lauma Pretkalnina, Sophie Prévost, Prokopis Prokopidis, Adam Przepiórkowski, Tiina Puolakainen, Sampo Pyysalo, Andriela Rääbis, Alexandre Rademaker, Loganathan Ramasamy, Taraka Rama, Carlos Ramisch, Vinit Ravishankar, Livy Real, Siva Reddy, Georg Rehm, Michael Rießler, Larissa Rinaldi, Laura Rituma, Luisa Rocha, Mykhailo Romanenko, Rudolf Rosa, Davide Rovati, Valentin Roșca, Olga Rudina, Shoval Sadde, Shadi Saleh, Tanja Samardžić, Stephanie Samson, Manuela Sanguinetti, Baiba Saulīte, Yanin Sawanakunanon, Nathan Schneider, Sebastian Schuster, Djamé Seddah, Wolfgang Seeker, Mojgan Seraji, Mo Shen, Atsuko Shimada, Muh Shohibussirri, Dmitry Sichinava, Natalia Silveira, Maria Simi, Radu Simionescu, Katalin Simkó, Mária Šimková, Kiril Simov, Aaron Smith, Isabela Soares-Bastos, Antonio Stella, Milan Straka, Jana Strnadová, Alane Suhr, Umut Sulubacak, Zsolt Szántó, Dima Taji, Yuta Takahashi, Takaaki Tanaka, Isabelle Tellier, Trond Trosterud, Anna Trukhina, Reut Tsarfaty, Francis Tyers, Sumire Uematsu, Zdeňka Urešová, Larraitz Uria, Hans Uszkoreit, Sowmya Vajjala, Daniel van Niekerk, Gertjan van Noord, Viktor Varga, Veronika Vincze, Lars Wallin, Jonathan North Washington, Seyi Williams, Mats Wirén, Tsegay Woldemariam, Tak-sum Wong, Chunxiao Yan, Marat M. Yavrumyan, Zhuoran Yu, Zdeněk Žabokrtský, Amir Zeldes, Daniel Zeman, Manying Zhang, and Hanzhi Zhu. 2018. Universal dependencies 2.2. LINDAT/CLARIN digital library at the Institute of Formal and Applied Linguistics (ÚFAL), Faculty of Mathematics and Physics, Charles University. http://hdl.handle.net/11234/12837.

Joakim Nivre, Marie-Catherine de Marneffe, Filip Ginter, Yoav Goldberg, Jan Hajič, Christopher Manning, Ryan McDonald, Slav Petrov, Sampo Pyysalo, Natalia Silveira, Reut Tsarfaty, and Daniel Zeman. 2016. Universal Dependencies v1: A multilingual treebank collection. In Proceedings of the 10th International Conference on Language Resources and Evaluation (LREC 2016). European Language Resources Association, Portorož, Slovenia, pages 1659-1666. http://www.lrecconf.org/proceedings/lrec2016/summaries/348.html.

Joakim Nivre and Chiao-Ting Fang. 2017. Universal dependency evaluation. In Proceedings of the NoDaLiDa 2017 Workshop on Universal Dependencies (UDW 2017). pages 86-95. 
Joakim Nivre, Johan Hall, Sandra Kübler, Ryan McDonald, Jens Nilsson, Sebastian Riedel, and Deniz Yuret. 2007. The CoNLL 2007 shared task on dependency parsing. In Proceedings of the CoNLL Shared Task Session of EMNLP-CoNLL 2007. Association for Computational Linguistics, pages 915-932. http://www.aclweb.org/anthology/D/D07/D071.pdf\#page $=949$.

Berkay Furkan Önder, Can Gümeli, and Deniz Yuret. 2018. SParse: Koç University graph-based parsing system for the CoNLL 2018 shared task. In Proceedings of the CoNLL 2018 Shared Task: Multilingual Parsing from Raw Text to Universal Dependencies. Association for Computational Linguistics.

Şaziye Betül Özateş, Arzucan Özgür, Tunga Güngör, and Balkız Öztürk. 2018. A morphology-based representation model for LSTM-based dependency parsing of agglutinative languages. In Proceedings of the CoNLL 2018 Shared Task: Multilingual Parsing from Raw Text to Universal Dependencies. Association for Computational Linguistics.

Martin Popel, Zdeněk Žabokrtský, and Martin Vojtek. 2017. Udapi: Universal API for universal dependencies. In NoDaLiDa 2017 Workshop on Universal Dependencies. Göteborgs universitet, Göteborg, Sweden, pages 96101. http://aclweb.org/anthology/W/W17/W170412.pdf.

Martin Potthast, Tim Gollub, Francisco Rangel, Paolo Rosso, Efstathios Stamatatos, and Benno Stein. 2014. Improving the reproducibility of PAN's shared tasks: Plagiarism detection, author identification, and author profiling. In Evangelos Kanoulas, Mihai Lupu, Paul Clough, Mark Sanderson, Mark Hall, Allan Hanbury, and Elaine Toms, editors, Information Access Evaluation meets Multilinguality, Multimodality, and Visualization. 5th International Conference of the CLEF Initiative (CLEF 14). Springer, Berlin Heidelberg New York, pages 268-299. https://doi.org/10.1007/978-3-31911382-1_22.

Peng Qi, Timothy Dozat, Yuhao Zhang, and Christopher D. Manning. 2018. Universal dependency parsing from scratch. In Proceedings of the CoNLL 2018 Shared Task: Multilingual Parsing from Raw Text to Universal Dependencies. Association for Computational Linguistics.

Rudolf Rosa and David Mareček. 2018. CUNI x-ling: Parsing under-resourced languages in CoNLL 2018 UD shared task. In Proceedings of the CoNLL 2018 Shared Task: Multilingual Parsing from Raw Text to Universal Dependencies. Association for Computational Linguistics.

Piotr Rybak and Alina Wróblewska. 2018. Semisupervised neural system for tagging, parsing and lematization. In Proceedings of the CoNLL 2018 Shared Task: Multilingual Parsing from Raw Text to
Universal Dependencies. Association for Computational Linguistics.

Amit Seker, Amir More, and Reut Tsarfaty. 2018. Universal morpho-syntactic parsing and the contribution of lexica: Analyzing the ONLP submission to the CoNLL 2018 shared task. In Proceedings of the CoNLL 2018 Shared Task: Multilingual Parsing from Raw Text to Universal Dependencies. Association for Computational Linguistics.

Aaron Smith, Bernd Bohnet, Miryam de Lhoneux, Joakim Nivre, Yan Shao, and Sara Stymne. 2018. 82 treebanks, 34 models: Universal dependency parsing with multi-treebank models. In Proceedings of the CoNLL 2018 Shared Task: Multilingual Parsing from Raw Text to Universal Dependencies. Association for Computational Linguistics.

Milan Straka. 2018. UDPipe 2.0 prototype at CoNLL 2018 UD shared task. In Proceedings of the CoNLL 2018 Shared Task: Multilingual Parsing from Raw Text to Universal Dependencies. Association for Computational Linguistics.

Milan Straka, Jan Hajič, and Jana Straková. 2016. UDPipe: trainable pipeline for processing CoNLL-U files performing tokenization, morphological analysis, POS tagging and parsing. In Proceedings of the 10th International Conference on Language Resources and Evaluation (LREC 2016). European Language Resources Association, Portorož, Slovenia.

Milan Straka and Jana Straková. 2017. Tokenizing, POS tagging, lemmatizing and parsing UD 2.0 with UDPipe. In Proceedings of the CoNLL 2017 Shared Task: Multilingual Parsing from Raw Text to Universal Dependencies. Association for Computational Linguistics.

Jörg Tiedemann. 2012. Parallel data, tools and interfaces in OPUS. In Nicoletta Calzolari (Conference Chair), Khalid Choukri, Thierry Declerck, Mehmet Ugur Dogan, Bente Maegaard, Joseph Mariani, Jan Odijk, and Stelios Piperidis, editors, Proceedings of the Eight International Conference on Language Resources and Evaluation (LREC'12). European Language Resources Association (ELRA), Istanbul, Turkey.

Hui Wan, Tahira Naseem, Young-Suk Lee, Vittorio Castelli, and Miguel Ballesteros. 2018. IBM Research at the CoNLL 2018 shared task on multilingual parsing. In Proceedings of the CoNLL 2018 Shared Task: Multilingual Parsing from Raw Text to Universal Dependencies. Association for Computational Linguistics.

Yingting Wu, Hai Zhao, and Jia-Jun Tong. 2018. Multilingual universal dependency parsing from raw text with low-resource language enhancement. In Proceedings of the CoNLL 2018 Shared Task: Multilingual Parsing from Raw Text to Universal Dependencies. Association for Computational Linguistics. 
Daniel Zeman, Martin Popel, Milan Straka, Jan Hajič, Joakim Nivre, Filip Ginter, Juhani Luotolahti, Sampo Pyysalo, Slav Petrov, Martin Potthast, Francis Tyers, Elena Badmaeva, Memduh Gökırmak, Anna Nedoluzhko, Silvie Cinková, Jan Hajič jr., Jaroslava Hlaváčová, Václava Kettnerová, Zdeňka Urešová, Jenna Kanerva, Stina Ojala, Anna Missilä, Christopher Manning, Sebastian Schuster, Siva Reddy, Dima Taji, Nizar Habash, Herman Leung, Marie-Catherine de Marneffe, Manuela Sanguinetti, Maria Simi, Hiroshi Kanayama, Valeria de Paiva, Kira Droganova, Héctor Martínez Alonso, Çağrı Çöltekin, Umut Sulubacak, Hans Uszkoreit, Vivien Macketanz, Aljoscha Burchardt, Kim Harris, Katrin Marheinecke, Georg Rehm, Tolga Kayadelen, Mohammed Attia, Ali Elkahky, Zhuoran Yu, Emily Pitler, Saran Lertpradit, Michael Mandl, Jesse Kirchner, Hector Fernandez Alcalde, Jana Strnadova, Esha Banerjee, Ruli Manurung, Antonio Stella, Atsuko Shimada, Sookyoung Kwak, Gustavo Mendonça, Tatiana Lando, Rattima Nitisaroj, and Josie Li. 2017. Conll 2017 shared task: Multilingual parsing from raw text to universal dependencies. In Proceedings of the CoNLL 2017 Shared Task: Multilingual Parsing from Raw Text to Universal Dependencies. Association for Computational Linguistics, Vancouver, Canada, pages 1-19. http://www.aclweb.org/anthology/K17-3001. 